\title{
DIFFUSE INTERFACE SURFACE TENSION MODELS IN AN EXPANDING FLOW*
}

\author{
WANGYI LIU ${ }^{\dagger}$, ANDREA BERTOZZI ${ }^{\ddagger}$, AND THEODORE KOLOKOLNIKOV ${ }^{\S}$
}

To Dave Levermore on his 60th birthday with friendship and appreciation

\begin{abstract}
We consider a diffusive interface surface tension model under compressible flow. The equation of interest is the Cahn-Hilliard or Allen-Cahn equation with advection by a non-divergence free velocity field. These are two reduced models which show important properties of the full-scale surface tension model. We prove that both model problems are well-posed. We are especially interested in the behavior of solutions with respect to droplet breakup phenomena. Numerical simulations of 1,2, and 3D all illustrate that the Cahn-Hilliard model is much more effective for droplet breakup. Using asymptotic methods we correctly predict the breakup condition for the Cahn-Hilliard model. Moreover, we prove that the Allen-Cahn model will not break up under certain circumstances due to a maximum principle.
\end{abstract}

Key words. Diffuse interface, surface tension, Cahn-Hilliard equation, numerical simulation.

AMS subject classifications. 76T10, 35B32, 35B50.

\section{Introduction}

We consider the following three models for studying the influence of flow field and surface tension on droplet breakup phenomena: the advective Cahn-Hilliard equation

$$
u_{t}+\nabla \cdot(u \vec{V})=\Delta K(u)
$$

the advective Allen-Cahn equation

$$
u_{t}+\nabla \cdot(u \vec{V})=-K(u)
$$

and the advective Allen-Cahn equation with mass conservation, or the nonlocal advective Allen-Cahn equation

$$
u_{t}+\nabla \cdot(u \vec{V})=-K(u)+\lambda u
$$

In these models, $\vec{V}$ is a prescribed velocity field and

$$
K(u)=-\epsilon \Delta u+\frac{1}{\epsilon} f(u) .
$$

The variable $\lambda$ is chosen so that $\int_{\Omega} u$ is a constant $M$. Or, if we ignore the boundary term,

$$
\lambda=\frac{\int_{\Omega} K(u)}{M}=\frac{1}{\epsilon} \frac{\int_{\Omega} f(u)}{M} .
$$

In full surface tension problems, the $\vec{V}$ is obtained via other equations, but the proposed model problem is simplified by having a prescribed $\vec{V}$. We consider these

\footnotetext{
*Received: November 22, 2010; accepted (in revised version): September 4, 2011.

${ }^{\dagger}$ Department of Mathematics, University of California Los Angeles, Los Angeles, California, USA (bobbyliu@math.ucla.edu).

${ }^{\ddagger}$ Department of Mathematics, University of California Los Angeles, Los Angeles, California, USA (bertozzi@math.ucla.edu).

$\S$ Department of Mathematics and Statistics, Dalhousie University, Halifax, Nova Scotia, Canada (tkolokol@mathstat.dal.ca).
} 
equations in order to understand their properties, which can be applied to coupled full problems. A comparison of these simplified models has not been studied in the literature and provides important insight on the behavior of the full problem. In this section we review some full surface tension models and discuss how the model problem is derived from them.

1.1. Different surface tension models. There are many numerical methods that deal with surface tension in two-phase fluids. This problem is known for its computational stiffness; it contains two different time scales, the small surface tension time scale, and the convection time scale. Three main algorithms exist for twophase fluids. The front tracking method tracks the interface explicitly, yet requires extensive processing when the interface splits and merges. Since droplet breakup mainly involves merging and splitting of the interface, it is unsuitable for the need. The level-set algorithm uses a implicit surface function to track the boundary. The diffusive interface algorithm uses a phase variable to describe the transition between materials. These algorithms, and many variants, have been studied both theoretically and numerically.

The basic level-set model for two immiscible fluids uses a function $\phi$, where $\phi=0$ denotes the boundary between the two fluids. For example, [34] combines the NavierStokes equation for two fluids with a force at the interface:

$$
\rho \frac{\partial \vec{V}}{\partial t}+(\vec{V} \cdot \nabla) \vec{V}=-\nabla p+\nabla \cdot(2 \nu D)-\tau \kappa(\phi) \nabla H(\phi)+f .
$$

This equation is then coupled with the level set equation for the interface:

$$
\phi_{t}+\nabla \cdot(\phi \vec{V})=0
$$

In this model, $\vec{V}$ is the velocity field, $D$ is the deformation tensor $\frac{1}{2}\left(\nabla \vec{V}+\nabla \vec{V}^{T}\right)-$ $\frac{1}{3} \nabla \vec{V} I, p$ is the pressure and $f$ denotes external force. These parameters are the same as the original Navier-Stokes model. $\kappa(\phi)=\nabla \cdot \frac{\nabla \phi}{|\nabla \phi|}$ is the curvature of the boundary, $\tau$ is the surface tension coefficient, and $H$ is the Heaviside function, or in the numerical implementation, a smoothed Heaviside function. Equation (1.6) is the Navier-Stokes equation with a surface tension term $\kappa \vec{n} \delta(d)$, where $\vec{n}$ is unit outward normal vector at the front, $d$ is normal distance to the front, and $\delta$ is the Dirac delta function. Recent models are designed to improve computational speed [35, 33]; see also [28, 13] for the compressible case.

The original Cahn-Hilliard equation [12] and the Allen-Cahn equation [3] are some of the most well-known dynamic models for diffuse interface dynamics associated with surface energies. The Cahn-Hilliard equation can be written as an $H^{-1}$ gradient descent for a Ginzburg-Landau free energy $E(u)$ :

$$
u_{t}=\Delta\left(\frac{\delta E(u)}{\delta u}\right)
$$

where

$$
E(u)=\int\left(\epsilon|\nabla u|^{2}+\frac{1}{\epsilon} g(u)\right),
$$

and $g(u)$ is a double-well potential that characterizes the two phases. It is normally taken as an even-order polynomial, for example

$$
g(u)=u^{2}(1-u)^{2},
$$


in which case $f(u)=g^{\prime}(u)=2 u(1-u)(1-2 u)$. The Allen-Cahn equation, on the other hand, is the $L^{2}$ gradient descent for the same energy:

$$
u_{t}=-\left(\frac{\delta E(u)}{\delta u}\right),
$$

Papers such as $[31,2,14]$ analyze the convergence and stability of the Cahn-Hilliard equation.

The combination of Cahn-Hilliard dynamics and fluid mechanics give rise to several related models for fluid interfaces. For example, the Navier-Stokes-Cahn-Hilliard model $[10,11,19,21,40,25,1,15]$ couples the incompressible fluid mechanics with a diffuse interface model. One variation of the model for an incompressible fluid (see $[21])$ is

$$
\begin{aligned}
\rho\left(\frac{\partial \vec{V}}{\partial t}+(\vec{V} \cdot \nabla) \vec{V}\right) & =-\nabla p+\nabla \cdot(2 \nu D)+f_{u}+f, \\
\nabla \cdot \vec{V} & =0 \\
\frac{\partial u}{\partial t}+\nabla \cdot(u \vec{V}) & =\Delta K(u) \\
K(u) & =\frac{\partial g(u)}{\partial u}-\epsilon^{2} \Delta u .
\end{aligned}
$$

The $f_{u}$ term in Equation (1.12) represent the influence of diffuse interface on the velocity field, and is different in different models. For example, in [19, 21] we have $f_{u}=-u \nabla K(u)$, and in [1] we have $f_{u}=\nabla \cdot\left(\frac{1}{2}|\nabla u|^{2} I-\nabla u \otimes \nabla u\right)$. Another variation of the above model to include compressible or quasi-incompressible fluids is found by replacing Equation (1.13) with

$$
\frac{\partial \rho}{\partial t}+\vec{V} \cdot \nabla \rho=\rho \nabla \cdot \vec{V},
$$

The additional advection term in Equation (1.14), compared to the original CahnHilliard, represents the mechanics of fluid flow, which is combined with the CahnHilliard type surface effect.

1.2. Model problem. The system equations (1.12-1.15) can be studied numerically, although it is difficult to prove properties of this system due to its coupled nature. We note that even simpler models have been considered for advection with interfacial tension $[24,27]$ in which the dynamics are driven by an Allen-Cahn type model rather than a Cahn- Hilliard model. A common tool in applied mathematics is that of developing reduced models for which one can prove rigorous results or perform asymptotic analysis, in order to understand the behavior of pieces of a more complex model. Such models may not describe the full physics of the real world problem, but can provide tremendous insight into the mathematical behavior and also provide both rigorous and asymptotic results that can be used in the development and testing of more complex numerical codes. Model problems have a long history in applied mathematics and we mention a few classical examples that have partially inspired our choice of problems. For example, the classical Kirchoff elliptical vortex for two dimensional fluids has been carefully studied under the influence of a fixed strain velocity field $[29,8]$, similar to the fixed field considered in this paper, although we focus on compressible examples as well as incompressible examples. A second example 
is a turbulent transport model that use a fixed background flow in order to derive renormalization formulas and other statistical properties associated with turbulent diffusion $[4,5]$. Another area of research in which interfacial dynamics is important is image processing. Many highly nonlinear PDEs have been proposed to address problems of denoising in image analysis. By combining these PDEs with a nonlinear transport term (Burgers flux), the second author and Greer were able to obtain some clear analytical and computational results on singularity formation for these nonlinear diffusion equations [16]. We note that the combined PDE is not directly used in image processing, although this model provides significant insight into the expected dynamics of more complex models that use these diffusions.

Following the ideas above, we choose to study the dynamics of the interface as defined by the Cahn-Hilliard or Allen-Cahn motion in a fixed prescribed velocity field. Since the interface is represented by $u$, we consider a reduced model of Equation (1.14) with a prescribed velocity field. This gives us the model problem Equation (1.1). We also use a different scaling for $K(u)$ compared to Equation (1.14), but the scaling is varied in the literature (e.g. $[40,25,26])$, and the properties we discuss will remain similar for all scalings. Model problem Equation (1.2) comes in a similar pattern, but it uses an Allen-Cahn based surface effect rather than a Cahn-Hilliard based surface effect. Equation (1.3) is based on Equation (1.2). If we integrate the original AllenCahn equation, we can see that it does not automatically conserve mass. Thus, an additional term $\lambda$ is often added to the equation for this reason [32]. We can add a similar term here, but we would like to add $\lambda u$ instead of $\lambda$ to keep $u$ localized. These models are also studied in the sharp interface limit $[24,26]$. The sharp interface limit for the Allen-Cahn equation is motion by mean curvature and the sharp interface limit for the Cahn-Hilliard is the Mullins-Sekerka flow. The analysis of these equations provide insight to their corresponding sharp interface limit problems.

Properties of $\vec{V}$ play an important role here. When $\vec{V}=0$ we obtain the original diffuse interface equation. Our main interest is when $\vec{V}$ is expanding, or general problems in which $\nabla \cdot \vec{V} \neq 0$. We note that the incompressible case is well studied, however the compressible case less so. For simplicity we choose the Neumann condition $\frac{\partial u}{\partial n}=0$ on $\partial \Omega$. In papers like $[11,21]$, the velocity field satisfies a Navier-Stokes equation, thus the velocity field $\vec{V}$ is divergent free. In our situation, $\vec{V}$ is not divergence free. The flow is expanding where $\nabla \cdot \vec{V}>0$ and contracting where $\nabla \cdot \vec{V}<0$.

In these advective equations, mass conservation acts in a different way due to boundary condition of the velocity field. For example, if we integrate Equation (1.1), we would have

$$
\left(\int_{\Omega} u\right)_{t}+\int_{\partial \Omega} u \vec{V} \cdot \vec{n}=\int_{\partial \Omega} \nabla K(u) \cdot \vec{n}
$$

Under a Neumann boundary condition, the right hand side becomes 0 . Only when we exert a no-flow condition $\vec{V} \cdot \vec{n}=0$ on the boundary can we have mass conservation. In fact, this no-flow condition would simplify many proofs below. We assume this is satisfied by having a small layer of $\vec{V}$ that vanishes near the boundary. We also assume $\vec{V}$ is smooth enough in the following arguments.

In the next section we analyze the basic property and droplet breakup condition of the advective Cahn-Hilliard equation. Section 3 analyzes the advective Allen-Cahn equation. Section 4 shows numerical simulation results for both models. 


\section{Property of the advective Cahn-Hilliard equation}

In this section we prove basic properties of the advective Cahn-Hilliard equation. We begin with the existence and uniqueness property of the equation, then move on to the analysis of the breakup condition.

2.1. Existence and uniqueness. The following existence and uniqueness theorem is similar to that of the original Cahn-Hilliard equation [36]. In the proof below and related arguments, the symbol $C$ denotes a generic constant.

THEOREM 2.1. If $g(u)$ in Equation (1.9) is a polynomial of order $2 p$, then for every given $u_{0}$ in $L^{2}(\Omega)$, the Equation (1.1) with $u(0)=u_{0}$ has a unique solution $u$ that belongs to $C\left([0, T] ; L^{2}(\Omega)\right) \cap L^{2}\left(0, T ; H_{0}^{2} \Omega\right) \cap L^{2 p}\left(0, T ; L^{2 p}(\Omega)\right), \forall T>0$.

The proof for this theorem follows the same steps as the Galerkin method for proving other equations like the Navier-Stokes equation and original Cahn-Hilliard equation, with the only difference in the a priori estimate; see $[37,36]$. We only present the different a priori estimates here.

The weak form of Equation (1.1) is

$$
\left(u^{\prime}(t), w\right)+\epsilon A(u, w)+B(\vec{V}, u, w)+\frac{1}{\epsilon}\left(f^{\prime}(u) \nabla u, \nabla w\right)=0, \forall w \in H^{2}(\Omega),
$$

where $A(u, w)=(\Delta u, \Delta w), B(\vec{V}, u, w)=\int_{\Omega} \nabla \cdot(u \vec{V}) w$.

Taking $w=u$ we have

$$
\frac{1}{2} \frac{d}{d t}|u|^{2}+\epsilon|\Delta u|^{2}+\frac{1}{\epsilon}\left(f^{\prime}(u) \nabla u, \nabla u\right)+\frac{1}{2}\left(|u|^{2}, \nabla \cdot \vec{V}\right)=0 .
$$

Since $f^{\prime}(s) \geq b_{2 p} s^{2 p-2}-C$,

$$
\frac{1}{2} \frac{d}{d t}|u|^{2}+\epsilon|\Delta u|^{2}+\frac{1}{\epsilon} \int_{\Omega}\left(b_{2 p} u^{2 p-2}|\nabla u|^{2}\right) \leq C|\nabla u|^{2}+\frac{C}{2}|u|^{2} .
$$

Thus we can get the upper bound for $u$ in $L^{2}\left(0, T ; H^{2}(\Omega)\right)$. To get the upper bound of $u$ in $L^{\infty}\left(0, T ; L^{2}(\Omega)\right)$, we see that

$$
\begin{aligned}
C|\nabla u|^{2} & \leq C|u|\|u\|_{H^{2}(\Omega)} \\
& \leq C|u|(|\Delta u|+M) \\
& \leq \frac{\epsilon}{2}|\Delta u|^{2}+C|u|^{2}+C M^{2},
\end{aligned}
$$

where $M=\int_{\Omega} u$ is the total mass. Thus,

$$
\frac{d}{d t}|u|^{2}+\epsilon|\Delta u|^{2}+\int_{\Omega}\left(b_{2 p} u^{2 p-2}|\nabla u|^{2}\right) \leq C|u|^{2}+C M^{2} .
$$

The rest of the proof is similar to $[37,36]$. By using the Gronwall inequality we get an upper bound for $u$ in $L^{\infty}\left(0, T ; L^{2}(\Omega)\right)$. This suffices to show the continuity and uniqueness.

2.2. Energy estimate. The original Cahn-Hilliard equation has an energy term that serves as a Lyapunov function:

$$
J(u)=\int_{\Omega} \frac{\epsilon}{2}|\nabla u|^{2}+\frac{1}{\epsilon} g(u) .
$$


This term can be estimated by multiplying $K(u)$ on both sides of Equation (1.1) and then integrating by parts. Following the same pattern, we get

$$
J(u)_{t}+(\nabla \cdot(u \vec{V}), K(u))=-|\nabla K(u)|^{2} .
$$

We can estimate the new term by

$$
(\nabla \cdot(u \vec{V}), K(u))=(\vec{V} \cdot \nabla u, K(u))+((\nabla \cdot \vec{V}) u, K(u)) .
$$

The first term,

$$
(\vec{V} \cdot \nabla u, K(u))=-\left(\nabla \cdot \vec{V}, \frac{\epsilon}{2}|\nabla u|^{2}+\frac{1}{\epsilon} g(u)\right)-\epsilon \int_{\Omega}(\nabla u)^{T} \nabla \vec{V} \nabla u,
$$

due to the fact that

$$
\begin{aligned}
\int_{\Omega} \vec{V} \cdot \nabla u \Delta u & =\int_{\Omega} \vec{V} \cdot\left(\nabla \cdot(\nabla u \otimes \nabla u)-\frac{1}{2} \nabla\left(|\nabla u|^{2}\right)\right) \\
& =\int_{\Omega}-\nabla \vec{V}:(\nabla u \otimes \nabla u)+\frac{1}{2} \nabla \cdot \vec{V}|\nabla u|^{2} \\
& =\int_{\Omega}-(\nabla u)^{T} \nabla \vec{V} \nabla u+\frac{1}{2} \nabla \cdot \vec{V}|\nabla u|^{2} .
\end{aligned}
$$

The right-hand side of Equation (2.9) is bounded from below by $-2\|\nabla \vec{V}\|_{L^{\infty}} J(u)$.

The second term of Equation (2.8) is bounded by

$$
\begin{aligned}
((\nabla \cdot \vec{V}) u, K(u)) & \geq-\epsilon\left(\frac{1}{2} \int u^{2} \Delta \nabla \cdot \vec{V}-\int|\nabla u|^{2} \nabla \cdot \vec{V}\right)-\frac{\|\nabla \cdot \vec{V}\|_{L^{\infty}}}{\epsilon} \int|u f(u)| \\
& \geq-C\left(J(u)+|u|_{2}^{2}\right) .
\end{aligned}
$$

Putting everything together, we have

$$
J(u)_{t} \leq C\left(J(U)+|u|_{2}^{2}\right),
$$

which, using Gronwall's inequality and the bound of $|u|_{2}^{2}$ above, gives

$$
J(u)_{t} \leq \exp (C t) J\left(u_{0}\right)+C .
$$

We can see that the energy of $u$ is bounded at every finite time interval $[0, T]$, and increases at most exponentially.

2.3. Droplet breakup. When the the external flow field is sufficiently large, the advective Cahn-Hilliard model exhibits droplet breakup as illustrated in Figure 2.1. Similar phenomena have been observed in numerous reaction-diffusion systems; see for example [30, 22], [20] and references therein. In this section we perform a detailed study of the breakup phenomenon for the advective Cahn-Hilliard model in one dimension. We recall the one dimensional case of Equation (1.1):

$$
u_{t}+(V(x) u)_{x}=K_{x x} ; \quad K=-\varepsilon u^{\prime \prime}+\frac{1}{\varepsilon} f(u) .
$$

We choose a specific form of $f(u)$ in our discussion:

$$
f(u)=2 u(1-u)(1-2 u) .
$$

Other forms of $f(u)$ follow a similar discussion. In [30], Nishiura and Ueyema proposed a set of conditions for the occurrence of self-replication in reaction-diffusion models. Roughly stated, they are: 
1. The disappearance of the steady state due to a fold-point (or saddle-node) bifurcation.

2. The existence of the so-called dimple-eigenfunction at the threshold, which is responsible for the initiation of the breakup process.

3. The steady state is stable on one side of the fold point and is unstable on the other.

The importance of these conditions is that the breakup of a droplet can be understood in terms of the analysis of the steady state solution of Equation (2.14) which satisfies

$$
(V(x) u)_{x}=K_{x x}, \quad K=-\varepsilon u^{\prime \prime}+\frac{1}{\varepsilon} f(u) .
$$

The breakup analysis for Equation (2.14) is very similar to [22], where the Brusselator and other reaction-diffusion systems having mesa-type structures were shown to exhibit self-replication. For simplicity, we will only consider a special case

$$
V(x)=\frac{V_{0}}{\varepsilon} x,
$$

and get the following asymptotic result:

RESUlt 2.2. Consider Equation (2.14) in the limit $\varepsilon \ll 1$, with $V(x)$ given by Equation (2.17), and with even initial conditions for $u$. For a given mass $M=\int_{-\infty}^{\infty} u d x$, let

$$
V_{c}=\frac{V_{c 0}}{M^{2}}
$$

where $V_{c 0} \approx 1.326$ is a constant whose precise value is given below in Equation (2.31). If $V<V_{c}$ then there exists a steady state $u(x, t)=u(x)$ in the form of a droplet. If $V>V_{c}$, no such steady state exists. As $V$ is slowly increased past $V_{c}$, the droplet will split in the middle and breakup into two droplets.

The derivation of this result consists of an analytic verification of the NishiuraUeyema Conditions 1 and 2. Due to space limitations, we omit the verification of Condition 3 but refer the reader to [22] where Condition 3 is proved for a similar model.

Verification of Nishiura-Ueyema Condition 1. We seek a steady state solution $u(x)$ which is even. It then follows that $K$ is also even and, upon integrating Equation $(2.16)$ on the interval $[0, x]$, we obtain

$$
K_{x}=\frac{V_{0}}{\varepsilon} x u .
$$

We now change variables $K=\frac{1}{\varepsilon} w$ to obtain a system

$$
w_{x}=V_{0} x u ; \quad-\varepsilon^{2} u_{x x}+f(u)=w .
$$

Since we assumed that $u$ is even, we consider only the half-line $x \geq 0$; the boundary conditions become

$$
u^{\prime}(0)=0=u^{\prime}(\infty) ; \quad \int_{0}^{\infty} u=M / 2
$$



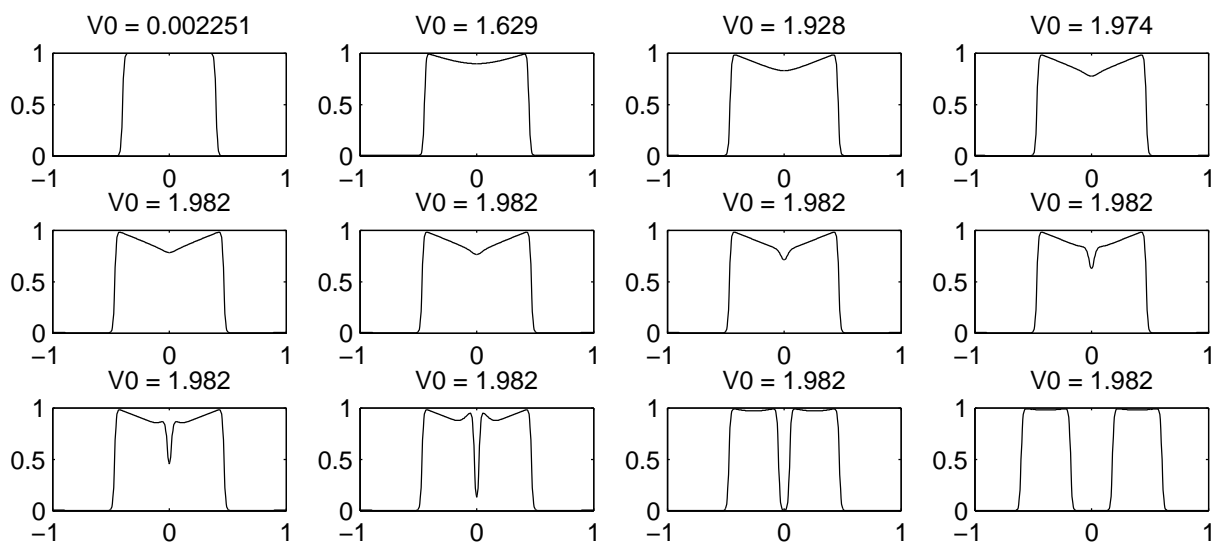

FIG. 2.1. Snapshots of temporal dynamics of Equation (2.16) with $V$ given by Equation (2.17). Here, $\varepsilon=0.01$ and the mass of the droplet is taken to be $M=\int_{-\infty}^{\infty} u d x=0.8$. The parameter $V_{0}$ is slowly increased in time according to the formula $V_{0}=0.001$.

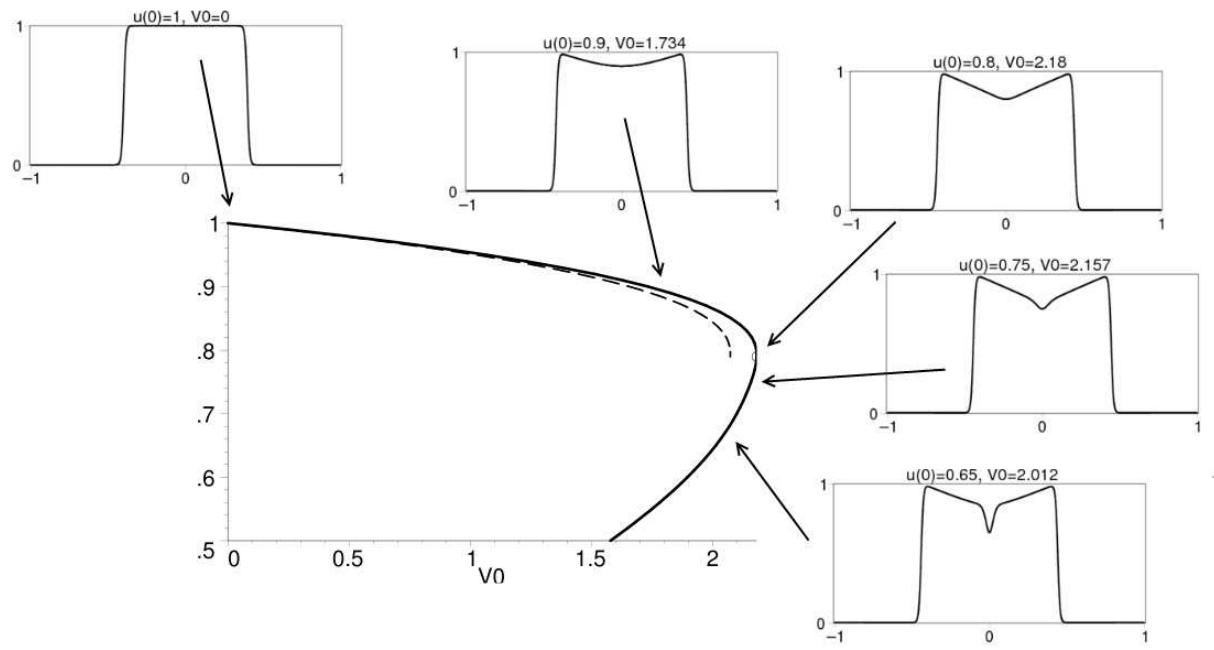

FIG. 2.2. The bifurcation structure of the the steady state Equation (2.19, 2.20) with $\varepsilon=$ $0.01, M=0.8 ; V_{0}$ is plotted vs. $u(0)$. The solid curve represents the numerical solution to the full system; the dotted curve is the asymptotic formula (2.29). The coordinates of the fold point are $u(0)=0.79, V_{0}=2.18$. The inserts show the profile of $u(x)$ for selected points along the bifurcation curve as indicated.

where $M$ is a given total mass of $u$. Since the time-dependent PDE Equation (2.14) conserves the mass of $u, M$ is also the initial mass of $u(x, t)$ at $t=0$.

We will construct a solution to Equation $(2.19,2.20)$ for which $u(x)$ has a sharp interface located at some position $x=l>0$ with $u \sim 0$ for $x>l$. Some typical such profiles for $u(x)$ are shown in Figure 2.2. Such a solution has a transition layer consisting of the interface near $x=l$ and an outer region to the left of $x=l$. In the 
transition layer, we rescale the space variable

$$
x=l+\varepsilon y ; \quad u(x)=U(y) ; \quad w(x)=W(y)
$$

to obtain

$$
W_{y} \sim \varepsilon^{2} V_{0} y U
$$

To leading order we have $W_{y} \sim 0$, so that $W \sim W_{0}$ is constant. We then obtain an ODE for $U$ :

$$
U_{y y}+f(U)-W_{0}=0 .
$$

The interface corresponds to a heteroclinic orbit of the ODE (2.21) which connects the two saddle equilibria of Equation (2.21). Such a heteroclinic connection exists if and only if $\int_{U_{-}}^{U_{+}}\left[f(U)-W_{0}\right] d U=0$, where $U_{ \pm}$are the equilibria points that satisfy $f\left(U_{ \pm}\right)-$ $W_{0}=0$ with $U_{+} \neq U_{-}$. Using $f(u)=2 u(1-u)(1-2 u)$, this yields $U_{+}=1, U_{-}=0$, and $W_{0}=0$; the explicit solution for $U(y)$ is then given by

$$
U(y)=\frac{1}{2}(1-\tanh (y / \sqrt{2})),
$$

with

$$
U(+\infty)=0 ; \quad U(-\infty)=1 .
$$

In the outer region away from the interface, to leading order we have

$$
f(u) \sim w, \quad 0 \leq x<l .
$$

Substituting Equation (2.22) into Equation (2.19) we obtain

$$
\frac{d u}{d x}=x V_{0} \frac{u}{f^{\prime}(u)} ; \quad u(l)=1 .
$$

The boundary condition is obtained from matching to the outer solution, $u(l)=$ $U(-\infty)=1$. The solution to Equation (2.23) is given by

$$
\frac{V_{0}}{2} x^{2}=\int_{u_{0}}^{u} \frac{f^{\prime}(s)}{s} d s ; x<l,
$$

where $u_{0}=u(0)$. Thus we obtain the following relationship between $l$ and $u_{0}$,

$$
\frac{V_{0}}{2} l^{2}=G\left(u_{0}\right),
$$

where

$$
G\left(u_{0}\right):=\int_{u_{0}}^{1} \frac{f^{\prime}(s)}{s} d s=-6 u_{0}^{2}+12 u_{0}-2 \ln u_{0}-6 .
$$

It remains to relate $l$ to $M$. Since $u \sim 0$ to the right of the interface, the mass of $u$ is asymptotically given by

$$
\frac{M}{2} \sim \int_{0}^{l} u(x) d x
$$




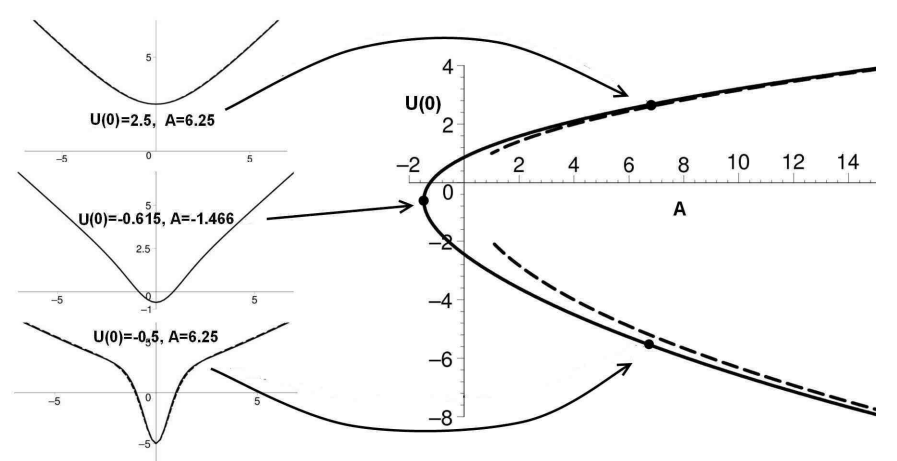

FIG. 2.3. Right side: Bifurcation diagram A vs. U(0) for the core problem (2.41). The solid curve is the numerical solution to Equation (2.41); dashed lines represent the asymptotics for large $A$ as given by equations (2.43,2.42). Left: the solution profiles with $U(0), A$ as indicated.

where we ignored the $O(\varepsilon)$ contribution to the mass from the interface. Writing Equation (2.24) as

$$
x^{2}=\frac{2}{V_{0}}\left(G\left(u_{0}\right)-G(u)\right)
$$

and substituting into Equation (2.26) we obtain

$$
\begin{aligned}
\frac{M}{2} & \sim\left(\frac{2}{V_{0}}\right)^{1 / 2} \int_{u_{0}}^{1} u \frac{d}{d u} \sqrt{G\left(u_{0}\right)-G(u)} d u \\
& \sim\left(\frac{2}{V_{0}}\right)^{1 / 2}\left\{\sqrt{G\left(u_{0}\right)}-\int_{u_{0}}^{1} \sqrt{G\left(u_{0}\right)-G(u)} d u\right\},
\end{aligned}
$$

so that

$$
V_{0} \sim \frac{1}{M^{2}} 8\left(\sqrt{G\left(u_{0}\right)}-\int_{u_{0}}^{1} \sqrt{G\left(u_{0}\right)-G(u)} d u\right)^{2} .
$$

Next, note that $G(1)=0$ and $G^{\prime}\left(u_{0}\right)=-f^{\prime}\left(u_{0}\right) / u_{0}$; in particular $G\left(u_{0}\right)$ attains a maximum at $u_{m}$ which satisfies $f^{\prime}\left(u_{m}\right)=0$ :

$$
u_{m}:=\frac{3+\sqrt{3}}{6}=0.78868
$$

It follows that the solution to the outer problem (2.23) only exists if $u_{m}<u(0)<1$. In terms of $M$, the critical threshold for existence is obtained by substituting $u_{0}=u_{m}$ into Equation (2.29); namely $V_{c}=\frac{V_{c 0}}{M^{2}}$ where the constant $V_{c 0}$ is given by

$$
V_{c 0}:=8\left(\sqrt{G\left(u_{m}\right)}-\int_{u_{m}}^{1} \sqrt{G\left(u_{m}\right)-G(u)} d u\right)^{2} \approx 1.32606 .
$$

This shows the existence of the fold-point for $V_{0}$ as given by Result 2.2 .

Verification of Nishiura-Ueyema Condition 2. Here, we follow closely an analogous derivation in [22]. The key is to demonstrate that when $V_{0}$ is close to the 
threshold value $V_{c}$, an additional boundary layer in the shape of an inverted spike forms at the center of the droplet. To see this, suppose that $V_{0}$ is sufficiently close to $V_{c}$ so that near $x=0$, we may expand

$$
u(x) \sim u_{m}+\delta u_{1}(x), \quad w \sim f\left(u_{m}\right)+\delta^{2} w_{1}+\ldots ; \quad \delta \ll 1 .
$$

The small parameter $\delta$ will be related to $\varepsilon$ below. The equation for $w_{1}$ then becomes

$$
\delta^{2} w_{1 x} \sim V_{0} x u_{m}
$$

so that

$$
w_{1}(x) \sim w_{1}(0)+\delta^{-2} \frac{V_{0} u_{m}}{2} x^{2} .
$$

The consistency condition for Equation (2.33) is that $x / \delta \ll \infty$; this will be satisfied below. We now expand in Taylor series,

$$
f(u)-w \sim-w_{1}(0) \delta^{2}-\frac{V_{0} u_{m}}{2} x^{2}+u_{1}^{2} \frac{f^{\prime \prime}\left(u_{m}\right)}{2} \delta^{2},
$$

where we recall that $f^{\prime}\left(u_{m}\right)=0$. Substituting Equations $(2.33,2.34)$ into Equation (2.19) we obtain

$$
\varepsilon^{2} u_{1 x x}-u_{1}^{2} \frac{f^{\prime \prime}\left(u_{m}\right)}{2} \delta^{2}+w_{1}(0) \delta^{2}+\frac{V_{0} u_{m}}{2} x^{2}=0 .
$$

To determine the right scaling for $\delta$, rescale

$$
x=\alpha z, u_{1}(x)=U(z)
$$

so that Equation (2.35) becomes

$$
U_{z z}-\left(\frac{f^{\prime \prime}\left(u_{m}\right)}{2} \frac{\delta^{2} \alpha^{2}}{\varepsilon^{2}}\right) U^{2}+\left(\frac{V_{0} u_{m}}{2} \frac{\alpha^{4}}{\varepsilon^{2}}\right) z^{2}+w_{1}(0) \frac{\delta^{2}}{\varepsilon^{2}} \alpha^{2}=0 .
$$

We now choose $\alpha, \delta$ so that Equation (2.36) becomes

$$
U_{z z}=U^{2}-z^{2}-A
$$

i.e.

$$
\begin{gathered}
\alpha:=\varepsilon^{1 / 2}\left(\frac{V_{0} u_{m}}{2}\right)^{-1 / 4} ; \quad \delta:=\varepsilon^{1 / 2}\left(\frac{V_{0} u_{m}}{2}\right)^{1 / 4}\left(\frac{f^{\prime \prime}\left(u_{m}\right)}{2}\right)^{-1 / 2}, \\
A:=\frac{2 w_{1}(0)}{f^{\prime \prime}\left(u_{m}\right)} .
\end{gathered}
$$

Matching with the outer solution, in the limit $z \rightarrow \infty$ we impose the boundary condition $u_{x x} \varepsilon^{2} \ll 1$, or $U_{z z} \sim 0$. Thus the boundary conditions for Equation (2.37) become

$$
U_{z}(0)=0 ; \quad U \sim z \text { as } z \rightarrow \infty .
$$

The Equations (2.37) and (2.40) together comprise the core problem which fully describes the growth of the inverted spike at the origin. The scaling $\alpha=O\left(\varepsilon^{1 / 2}\right)$ quantifies the width of the the core spike in terms of the $O(\varepsilon)$ interface width. This 
core problem is identical to the core problem for the Brusselator and other reactiondiffusion systems; we refer the reader to [22] for details. For convenience, we state the main result about Equations $(2.37,2.40)$ as derived in [22]:

Lemma 2.3 (From [22], Theorem 2). Consider the core problem

$$
U_{z z}=U^{2}-z^{2}-A ; \quad U_{z}(0)=0 ; \quad U \sim z \text { as } z \rightarrow \infty .
$$

There exists a constant $A_{c}$ such that Equation (2.41) has precisely two monotone solutions for $A>A_{c}$ and no monotone solutions when $A<A_{c}$.

When $A \gg 1$, Equation (2.41) admits two monotone solutions $U^{ \pm}(z)$ with the following uniform asymptotic expansions:

$$
\begin{aligned}
& U^{+}(z) \sim \sqrt{A+z^{2}}, \quad \text { with } \quad U^{+}(0) \sim \sqrt{A} \\
& U^{-}(z) \sim \sqrt{A+z^{2}}\left(1-3 \operatorname{sech}^{2}\left(\frac{A^{1 / 2} z}{\sqrt{2}}\right)\right), \quad \text { with } \quad U^{+}(0) \sim-2 \sqrt{A} .
\end{aligned}
$$

For any monotone solution of Equation (2.41), let $s=U(0)$ and consider the curve $A=A(s)$. Then $A(s)$ has a unique (minimum) critical point at $s=s_{c}, A=A_{c}$. Moreover, define

$$
\Phi(z)=\left.\frac{\partial U(z ; s)}{\partial s}\right|_{s=s_{c}}
$$

Then $\Phi(z)>0$ for all $z \geq 0$ and $\Phi \rightarrow 0$ as $z \rightarrow \infty$. Numerically, $A_{c}=-1.46638, s_{c}=$ -0.61512 .

The bifurcation diagram of $A$ vs. $U(0)$ and some steady states is given by Figure 2.3.

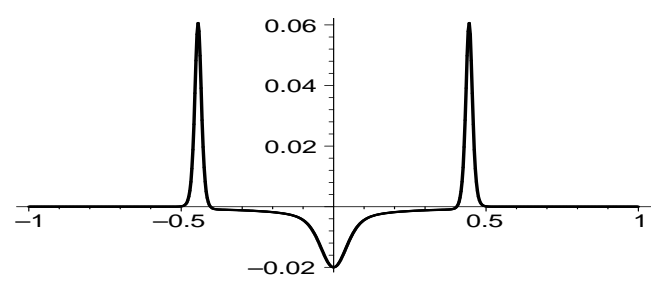

FIG. 2.4. The shape of the the eigenfunction corresponding to the zero eigenvalue at the fold point of the bifurcation diagram for Equation (2.19,2.20) with $\varepsilon=0.01, M=0.8$. The parameter $V_{0}=2.18$ is chosen to be at the fold point.

In particular, the profile $U^{-}$describes the shape of the finger within the boundary layer at the center of the droplet, which is responsible for the initiation of the splitting process. Similarly, as was shown in [22], the linearized problem at the fold point has a zero eigenvalue; the corresponding eigenfunction is given by $\phi=\partial u / \partial[u(0)]$. Moreover, $\int \phi=0$ due to mass conservation. As explained in [22], it follows from Lemma 2.3 that $\phi$ has precisely one positive root; its profile is shown in Figure 2.4. This proves that criterion 2 of Nishiura-Ueyema conditions is satisfied. This concludes the derivation of our result.

We use two methods to verify the droplet breakup condition from Result 2.2. We take $\varepsilon=0.01$ and we let $V_{0}$ be a slowly varying parameter in time, according to the 
formula $V_{0}=0.001 t$. Using the initial condition

$$
u(x, 0)=\frac{1}{2}\left(1-\tanh \left(\frac{|x|-0.4}{0.01 \sqrt{2}}\right)\right),
$$

we then numerically compute the solution to the full system (1.1). Droplet breakup is observed at about $t=1982$ or $V_{0} \approx 1.982$ as shown in Figure 2.1. The initial conditions Equation (2.44) correspond to $M=0.8$. The formula (2.18) for $V_{c}$ then yields $V_{c}=$ $\frac{1.32606}{0.8^{2}}=2.07$, which compares favorably with the numerical result.

Next, we computed the bifurcation diagram of the steady state Equations (2.19, $2.20)$; this is shown in Figure 2.2. To compute the diagram, we gradually changed $u(0)$ from 1 down to 0.5 ; then for each given $u(0)$, we used Maple's numerical boundary value problem solver to compute the corresponding value of $V_{0}$. In this way, the fold point was found at $u(0)=0.79$, with the corresponding $V_{0}=2.18$. This agrees very well with the asymptotic result $V_{c}=2.07$ as well as Equation (2.30), $u_{m}=0.7887$.

In higher dimensions, similar result can be deducted for the radially symmetric case. In fact, it is only necessary to modify Equation (2.16) slightly:

$$
\begin{aligned}
& \left(\frac{d}{d r}+(n-1) \frac{1}{r}\right)(V(r) u)=\left(\frac{d}{d r}+(n-1) \frac{1}{r}\right) K_{r} ; \\
& K=-\varepsilon\left(\frac{d}{d r}+(n-1) \frac{1}{r}\right) u_{r}+\frac{1}{\varepsilon} f(u) .
\end{aligned}
$$

The asymptotic solution would be the same as Equation (2.24), but the relationship between $l$ and $M$ become $M \int_{0}^{l} C r^{n-1} u(r) d r$ instead where $C$ is the volume of the $n-1$ dimensional unit sphere. Thus, the relationship Equation (2.18) becomes $V_{c}=\frac{V_{c 0}}{M^{2 / n}}$ in $n$ dimensions.

\section{Property of the advective Allen-Cahn equation}

In this section we prove the existence, uniqueness and maximum principles for the advective Allen-Cahn equation and the advective nonlocal Allen-Cahn equation. The maximum principle shows that the droplet breakup will not appear in many cases.

3.1. Existence and uniqueness. The existence and uniqueness for the advective Allen-Cahn equation can be done similarly to that of the advective CahnHilliard equation. However, a different method must be used for the advective nonlocal Allen-Cahn equation with mass conservation due to the extra nonlocal term. A semigroup method is used to show finite time existence of the solution, then a maximum principle analysis gives the bound of the $\lambda$ in the non-local term.

Theorem 3.1. For dimension $n=1,2,3$, if $g(u)$ in Equation (1.9) is a polynomial of order $2 p$, then Equations (1.2) and (1.3) with initial value $u_{0} \in W^{\frac{3}{2}, 2}(\Omega)$ have a unique solution $u \in C^{1}\left([0, T] ; C^{2}(\Omega)\right), \forall T>0$.

The proof contains two parts. The first part follows a similar process to that used in $[6,18]$, which involves using their following propositions.

Proposition 3.2. Consider the equation

$$
u_{t}=A u+N(u),
$$

where $A$ is the generator of a holomorphic semigroup $S(t)$ of bounded linear operators on a Banach space $X$. Suppose that $\|S(t)\| \leq M_{0}$ for some constant $M_{0}>0$ for all $t>$ 
0. Under these hypotheses the fractional powers $(-A)^{-\alpha}$ can be defined for $0 \leq \alpha<1$ and $(-A)^{\alpha}$ is a closed linear operator with domain $X_{\alpha}=$ Domain $\left((-A)^{\alpha}\right)$ dense in $X$. Let $N(u)$ be locally Lipschitz, i.e. for each bounded subset of $U$ there exists a constant $C_{U}$ such that

$$
\left\|N\left(u_{1}\right)-N\left(u_{2}\right)\right\| \leq C_{U}\left\|u_{1}-u_{2}\right\|_{\alpha}, \forall u, v \in U
$$

then given $u_{0} \in X$, there exists a finite time interval $[0, t)$ and a unique solution $u$ with $u(0)=u_{0}$ on the time interval and the solution can be continued uniquely on a maximal interval of existence $\left[0, T^{*}\right)$. Moreover, if $T^{*}<\infty$ then $\lim _{t \rightarrow T^{*}}\|u(t)\|_{\alpha}=\infty$.

Proposition 3.3. Assume $A$ and $N$ same as above, suppose $u$ is a solution of the equation on $(0, T]$, then if $\gamma<1, t \rightarrow u_{t}(t) \in X_{\gamma}$ is locally Hölder continuous for $t \in(0, T]$, with $\left\|u_{t}\right\|_{\alpha} \leq C t^{\alpha-\gamma-1}$.

Lemma 3.4. Assume $A$ and $N$ are as above. If $\|N(u)\| \leq C(t)\left(1+\|u\|_{\alpha}\right)$, then the unique solution exists for all times.

In Equation (1.3), we can take $A=\epsilon \Delta$ on the domain of $H^{2}(\Omega)$ functions with Neumann boundary condition, $1>\alpha>\frac{3}{4}, X=L^{2}(\Omega)$ and

$$
N(u)=-\nabla \cdot(u \vec{V})-\frac{1}{\epsilon} f(u)+\lambda u .
$$

We have $X_{\alpha} \supset W^{\frac{3}{2}, 2}(\Omega) \cap L^{\infty}(\Omega)$. Thus, we can estimate the three terms of $N\left(u_{1}\right)-$ $N\left(u_{2}\right)$ individually.

$$
\begin{aligned}
\left\|\nabla \cdot\left(u_{1} \vec{V}\right)-\nabla \cdot\left(u_{2} \vec{V}\right)\right\|_{L^{2}} & \leq\|\vec{V}\|_{L^{\infty}}\left\|\nabla u_{1}-\nabla u_{2}\right\|_{L^{2}}+\|\nabla \cdot \vec{V}\|_{L^{\infty}}\left\|u_{1}-u_{2}\right\|_{L^{2}} \\
& \leq C\left\|u_{1}-u_{2}\right\|_{H^{1}} \\
& \leq C\left\|u_{1}-u_{2}\right\|_{X^{\alpha}} .
\end{aligned}
$$

Since $f$ is a polynomial of order $2 p-1$ we have

$$
f\left(u_{1}\right)-f\left(u_{2}\right)=\left(u_{1}-u_{2}\right) h\left(u_{1}, u_{2}\right),
$$

where $h$ is a polynomial of order $2 p-2$.

$$
\begin{aligned}
\left\|f\left(u_{1}\right)-f\left(u_{2}\right)\right\|_{L^{2}} & \leq\left\|u_{1}-u_{2}\right\|_{L^{2}}\left\|h\left(u_{1}, u_{2}\right)\right\|_{L^{\infty}} \\
& \leq C\left\|u_{1}-u_{2}\right\|_{L^{2}}\left(\left\|u_{1}\right\|_{L^{\infty}}^{2 p-2}+\left\|u_{2}\right\|_{L^{\infty}}^{2 p-2}\right) \\
& \leq C\left\|u_{1}-u_{2}\right\|_{X^{\alpha}}\left(\left\|u_{1}\right\|_{X^{\alpha}}^{2 p-1}+\left\|u_{2}\right\|_{X^{\alpha}}^{2 p-1}\right),
\end{aligned}
$$

and

$$
\begin{aligned}
& \left\|u_{1} \int_{\Omega} f\left(u_{1}\right)-u_{2} \int_{\Omega} f\left(u_{2}\right)\right\|_{L^{2}} \\
\leq & \left\|u_{1}\right\|_{L^{2}}\left\|f\left(u_{1}\right)-f\left(u_{2}\right)\right\|_{L^{1}}+\left\|u_{1}-u_{2}\right\|\left\|_{L^{2}}\right\| f\left(u_{2}\right) \|_{L^{1}} \\
\leq & \left\|u_{1}-u_{2}\right\|_{L^{1}}\left\|u_{1}\right\|_{L^{2}}\left\|h\left(u_{1}, u_{2}\right)\right\|_{L^{\infty}}+C\left\|u_{1}-u_{2}\right\|_{L^{2}}\left\|u_{2}\right\|_{L^{2 p-1}}^{2 p-1} \\
\leq & C\left\|u_{1}-u_{2}\right\|_{X^{\alpha}}\left\|u_{1}\right\|_{L^{\infty}}\left(\left\|u_{1}\right\|_{L^{\infty}}^{2 p-2}+\left\|u_{2}\right\|_{L^{\infty}}^{2 p-2}\right)+C\left\|u_{1}-u_{2}\right\|_{X^{\alpha}}\left\|u_{2}\right\|_{L^{\infty}}^{2 p-1} \\
\leq & C\left\|u_{1}-u_{2}\right\|_{X^{\alpha}} \|\left(\left\|u_{1}\right\|_{X^{\alpha}}^{2 p-1}+\left\|u_{2}\right\|_{X^{\alpha}}^{2 p-1}\right) .
\end{aligned}
$$

We can apply Proposition 3.2 from here and get a unique solution in $u \in D(A)$. Then, since $\nabla u \in W^{1,2}(\Omega) \subset L^{6}(\Omega)$, we have $A u=N(u)-\frac{d u}{d t} \in L^{6}(\Omega)$. This implies $\nabla u \in$ 
$W^{1,6}(\Omega)$, which is Hölder continuous. This in turn shows $u \in C^{2+\delta}(\Omega)$ for some $\delta>0$. The local Lipschitz condition for Equation (1.2) is similar.

This proposition shows a maximum interval of existence $\left[0, T_{\max }\right)$ for the advective Allen-Cahn equations. To show global existence, we need maximum principle below to show a bound for $\lambda$ and $f(u)$, then we can directly apply Lemma 3.4 using the fact that

$$
\|\nabla \cdot(u \vec{V})\|_{L^{2}} \leq C|| u\left\|_{H^{1}} \leq C\right\| u \|_{X^{\alpha}} .
$$

3.2. Maximum principle analysis. The maximum principle-like analysis works only for the advective Allen-Cahn equation, since it is second-order and parabolic. The advective Cahn-Hilliard equation, on the other hand, is of fourthorder and thus does not possess the maximum principle.

THEOREM 3.5. For Equation (1.2) with any velocity field, or Equation (1.3) with expanding flow $\nabla \cdot \vec{V} \geq 0$, there exists a value $u_{M}$ such that, if initial value $u_{0}(x) \in$ $\left[0, u_{M}\right]$ in $\Omega$ and satisfies the condition of Theorem 3.1, then $u(x, t) \in\left[0, u_{M}\right]$ for all $t$. For Equation (1.3) with a general flow, $0 \leq u(x, t) \leq \max (\exp (-\inf (\nabla \cdot \vec{V}) t), 1) u_{M}$.

If we set $\hat{u}(x, t)=\exp ^{\xi t} u(x, t)$, then Equation (1.2) becomes

$$
\hat{u}_{t}=\exp ^{\xi t}\left(\epsilon \Delta u-\nabla u \cdot \vec{V}-u \nabla \cdot \vec{V}-\frac{1}{\epsilon} f(u)+\xi u\right),
$$

and Equation (1.3) becomes

$$
\hat{u}_{t}=\exp ^{\xi t}\left(\epsilon \Delta u-\nabla u \cdot \vec{V}-u \nabla \cdot \vec{V}-\frac{1}{\epsilon} f(u)-\lambda u+\xi u\right) .
$$

For the advective Allen-Cahn Equation (1.2), we can simply take $\xi$ close to 0 . Since $g(u)$ is a double-well potential, $f(u)<0$ when $u<0$. We can deduce that there is no interior negative minimum. Similarly, there is no interior maximum larger than 1. Thus, if the initial value is within $[0,1]$, so is the solution.

For the advective nonlocal Allen-Cahn Equation (1.3) it becomes a little more complicated. Within any time interval $[0, T], \lambda$ is bounded, so we can take a proper $\xi$ in Equation (3.10) to use the maximum principle. Thus, $\hat{u}$ has no negative minimum within any interval $(0, T]$. If initial value is nonnegative, so is the solution.

The positive side is more tricky. If $u$ takes its maximum value $u_{\max }$ on the interior and $\nabla \cdot \vec{V} \geq 0$, then at that point we have $\frac{1}{\epsilon} f\left(u_{\max }\right)+\lambda u_{\max }<0$. On the other hand, due to the definition of $\lambda$ Equation (1.5), we know that $\lambda=-\frac{1}{\epsilon} \frac{f(u)}{u}$ for some $u \in\left[0, u_{\max }\right]$. This means that $\frac{f\left(u_{\max }\right)}{u_{\max }}<\frac{f(u)}{u}$ for some $u \in\left[0, u_{\max }\right]$. Since $\frac{f(u)}{u}$ is an even-ordered polynomial, there exists an $u_{M}>0$ so that $\frac{f\left(u_{\max }\right)}{u_{\max }} \geq \frac{f(u)}{u}$ for all $u_{\max } \geq u_{M}$ and $u \leq u_{\max }$. Thus, if the initial value is smaller than $u_{M}$, so is the solution. For example, if we take double-well potential $g(u)=u^{2}(1-u)^{2}$, then $u_{M}=1.5$. For a general flow, we take $\xi=\inf (\nabla \cdot \vec{V})$ in Equation (3.10). With a similar analysis, $u \leq u_{M}$ when $\hat{u}$ takes its maximum, hence the result.

For the advective Cahn-Hilliard Equation (1.1), the maximum principle analysis does not work. In fact, there are cases when it fails: the solution becomes negative even when the initial value is not; see numerical results in Figure 4.5, Figure 4.13, and Figure 4.15 .

In the simple 1D case, we can show the following fact: 
THEOREM 3.6. If $u$ satisfies Equation (1.2) or (1.3), $u(x, 0) \geq 0$ and bounded, $u_{x}(x, 0) \leq 0$ on $\Omega$, and $V_{x x}(x) \geq 0$, then $u_{x}(x, t) \leq 0$ for all $t$.

Note that, if we expect a symmetric condition, i.e. $V$ is odd and $u$ is even, and $u(x, 0)$ takes its only maximum value at $x=0$, then $u_{x}(0, t)=0$ and we can apply this theorem on $\Omega \cap[0, \infty)$. Thus for any $t, u(x, t)$ takes the maximum value at $x=0$, and the droplet breakup does not occur.

To prove this, we see that Equation (1.2) leads to

$$
\left(u_{x}\right)_{t}=\left(u_{x}\right)_{x x}-V\left(u_{x}\right)_{x}-\left(2 V_{x}+f^{\prime}(u)\right) u_{x}-V_{x x} u,
$$

and Equation (1.3) leads to

$$
\left(u_{x}\right)_{t}=\left(u_{x}\right)_{x x}-V\left(u_{x}\right)_{x}-\left(2 V_{x}+f^{\prime}(u)+\lambda\right) u_{x}-V_{x x} u .
$$

Since $2 V_{x}+f^{\prime}(u)$ and $\lambda$ are bounded, $V_{x x} u \geq 0$, we can use a process similar to that above to show that no positive maximum can be achieved in the interior of $\Omega$. Thus $u_{x}(x, t) \leq 0$ for all $t$. When $V_{x x}$ is not nonnegative, breakup may occur. See Figure 4.11 and Figure 4.12 .

In higher dimensions, it is easy to consider the case of radially symmetric data. General results require a more detailed analysis, but this analysis is suffice to show that an Allen-Cahn type equation is unsuitable for the model of droplet breakup.

THEOREM 3.7. Suppose u satisfies Equation (1.2) or (1.3) on a n-dimensional sphere around $0, u(\vec{x}, 0) \geq 0$ bounded and radially symmetric, and $u_{r}(\vec{x}, 0) \leq 0$ on $\Omega$, where $u_{r}$ is the directional derivative of $u$ in the direction of $\vec{x}$. If $\vec{V}(\vec{x})=V(|\vec{x}|) \frac{\vec{x}}{|\vec{x}|}$ and $r^{2} V_{r r}(r)+(n-1) r V_{r}(r)-(n-1) V(r) \geq 0$ for any $r$, then $u_{r}(\vec{x}, t) \leq 0$ for all $t$.

We can prove this by taking $w=r^{n-1} u_{r}$, so that Equation (1.2) gives

$$
w_{t}=w_{r r}-\left(\frac{n-1}{r}-V\right) w_{r}-\left(2 V_{r}+f^{\prime}(u)\right) w-r^{n-3}\left(r^{2} V_{r r}+(n-1) r V_{r}-(n-1) V\right) u .
$$

Using the same method as that of $1 \mathrm{D}$ case, we can show that no positive maximum exist under given condition. Thus, $w \geq 0$ for all $\vec{x}$ and $t$, which is equivalent to $u_{r} \geq 0$. When $n=1$, the condition on $\vec{V}$ would be the same as in the 1D Theorem 3.6.

\section{Numerical simulation}

4.1. Algorithm. In this section we present numerical simulations in 1, 2, and 3D. We compare some of the results with the theory from previous sections. Specifically, we focus on different behaviors when the strength of the velocity field changes, and different droplet breakup conditions for different models. All of our numerical results are consistent with the theories in previous sections.

The Cahn-Hilliard equation poses numerical challenges due to the stiffness of both the 4th-order term and the nonlinear term. Thus many algorithms, both linear and nonlinear, have been proposed to solve it, for example the finite element method [7] and semi-implicit discretization $[38,39,9]$. In this paper we apply a simple semiimplicit splitting scheme [38] on the fourth-order term of the advective Cahn-Hilliard Equation (1.1). The equation can be written as

$$
\frac{u^{n+1}-u^{n}}{\Delta t}+\nabla \cdot\left(u^{n} \vec{V}\right)=-\Delta\left(\epsilon \Delta\left(A u^{n+1}+(1-A) u^{n}\right)-\frac{1}{\epsilon} f\left(u^{n}\right)\right),
$$




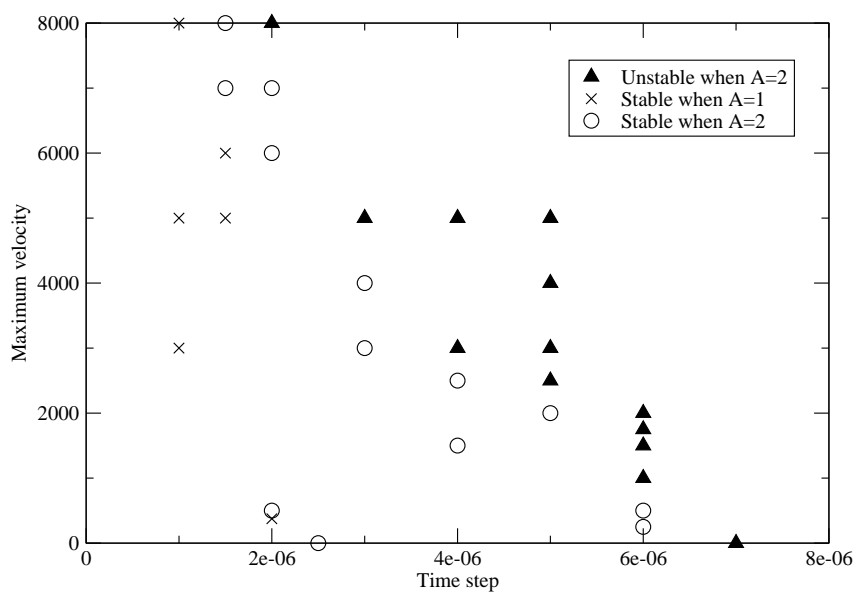

FIG. 4.1. The Stability graph for the 1D advective Cahn-Hilliard Equation (1.1) using the scheme in (4.1). The cross shape indicates the stable cases for both $A=1$ and $A=2$, the circle represents the stable case for $A=2$ but the unstable case for $A=1$, the triangle shape indicate unstable case for $A=2$. The $X$-axis and $Y$-axis represent the time step $\Delta t$ and the maximum value of velocity field $V_{\max }$ respectively.

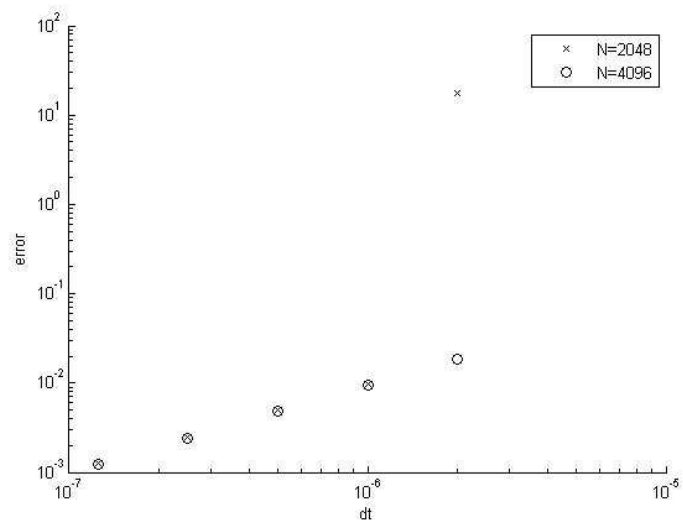

FIG. 4.2. The convergence graph for the $1 D$ advective Cahn-Hilliard Equation (1.1) using the scheme in (4.1). Error $=\left|u_{\Delta x, \Delta t}(t)-u_{\Delta x, \Delta t / 2}(t)\right|$. The $x$-axis is the value of $\Delta t . \Delta x$ is $10 / 2048$ for the crosses and 10/4096 for the circles. Note that the rightmost cross is an unstable situation. For $\Delta x=10 / 4096$ the instability will not be visible until a larger $t$. The error converges to 0 at order 1.

where the advection term is discretized by the upwind scheme. The parameter $A$ is chosen as 1 in the implementation. Equations (1.2) and (1.3) are discretized as

$$
\frac{u^{n+1}-u^{n}}{\Delta t}+\nabla \cdot\left(u^{n} \vec{V}\right)=\epsilon \Delta\left(A u^{n+1}+(1-A) u^{n}\right)-\frac{1}{\epsilon} f\left(u^{n}\right)
$$




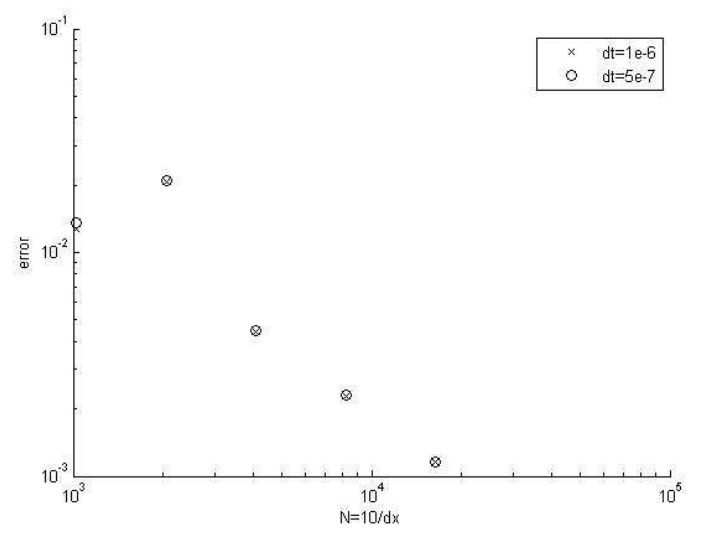

FIG. 4.3. The convergence graph for the $1 D$ advective Cahn-Hilliard Equation (1.1) using the scheme in (4.1). Error $=\left|u_{\Delta x, \Delta t}(t)-u_{\Delta x / 2, \Delta t}(t)\right|$. The $x$-axis is the value of $N=10 / \Delta x . \Delta t$ is $1 \times 10^{-6}$ for the crosses and $5 \times 10^{-7}$ for the circles. Note that the error converges to 0 at order 1 .
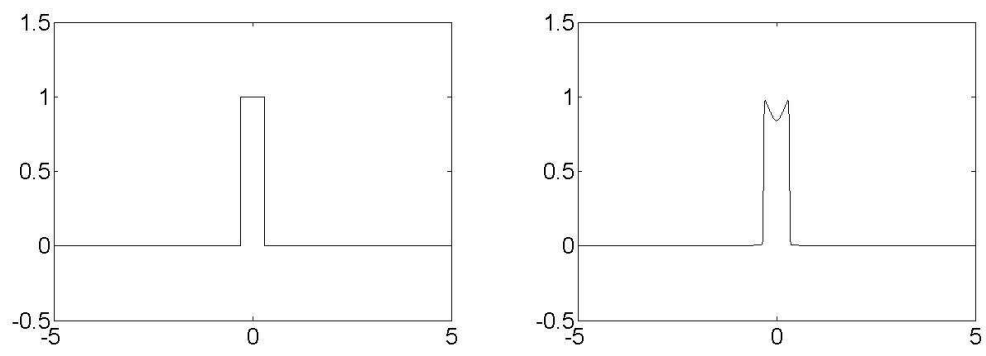

FIG. 4.4. The advective Cahn-Hilliard equation does not breakup with $V_{0}=400$, at time 0 and $20 \times 10^{-4}$ respectively.

and

$$
\frac{u^{n+1}-u^{n}}{\Delta t}+\nabla \cdot\left(u^{n} \vec{V}\right)=\epsilon \Delta\left(A u^{n+1}+(1-A) u^{n}\right)-\frac{1}{\epsilon} f\left(u^{n}\right)+\lambda u,
$$

respectively.

The stability condition now is related to $\vec{V}$ and the value of $A$. For example, the graph of stability of the advective Cahn-Hilliard equation related to time step $\Delta t$ and the maximum norm of $\vec{V}$ is shown in Figure 4.1. $\Delta x$ has some insubstantial effects on the stability, but not so much as a CFL condition would require. In fact, the coefficient of $u^{n+1}$ is $I+\Delta t \epsilon \Delta^{2}$, and is on the order of $(\Delta x)^{-4}$ when $\Delta x$ is small. This is of a higher order than the advective term $\vec{V} \cdot \nabla u^{n}$, thus providing the main constraint for stability. This stability condition with $V=0$ is consistent with similar results for the plain Cahn-Hilliard equations as in [17].Reference [9] shows that a scheme of this kind would have an error of $O(C \Delta t)$, but the constant $C$ would be very large. With the additional advection term, $C$ becomes related to $V_{\max }=\|\vec{V}\|_{L^{\infty}}$, and thus when $V_{\max }$ increases, a smaller time step would be required. Moreover, when $\vec{V}$ is not very large, the most important constraint on $\Delta t$ comes from the stability of the original 

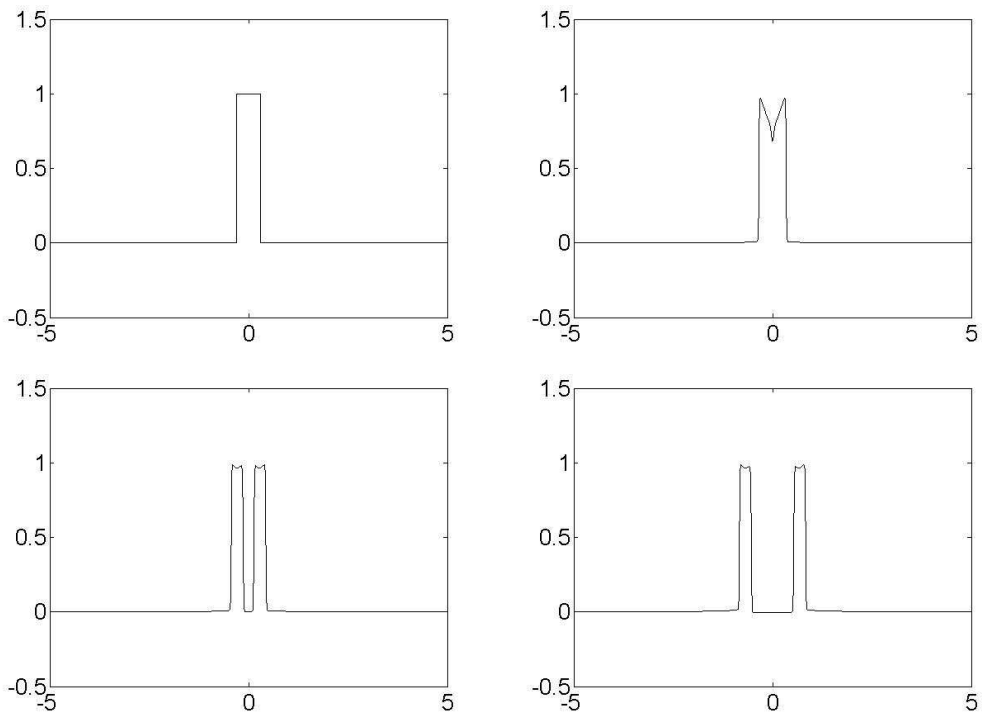

FIG. 4.5. The advective Cahn-Hilliard equation breakup with $V_{0}=600$, at time $t=0,5 \times 10^{-4}$, $15 \times 10^{-4}$, and $20 \times 10^{-4}$ respectively.

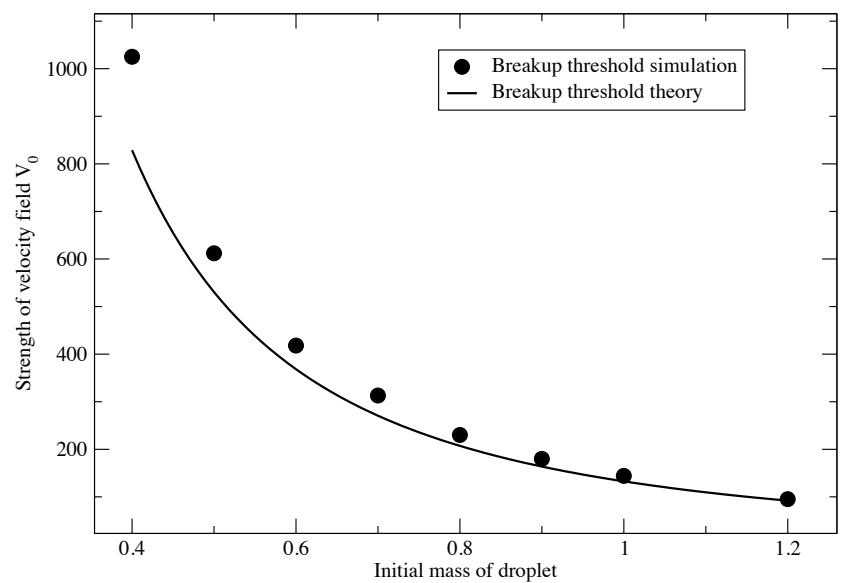

FIG. 4.6. Threshold for Cahn-Hilliard. Dots are simulation data, and the line is an inverse quadratic curve $V_{0} M^{2}=1.326$.

Cahn-Hilliard equation. Using $A=2$ instead of $A=1$ largely increases the maximum time step required for stability.

To get a clear view of the convergence result, we consider the limit of $\Delta x \rightarrow 0$ and $\Delta t \rightarrow 0$ individually and plot an example of the graphs of $\left|u_{\Delta x, \Delta t}(t)-u_{\Delta x, \Delta t / 2}(t)\right|$ 

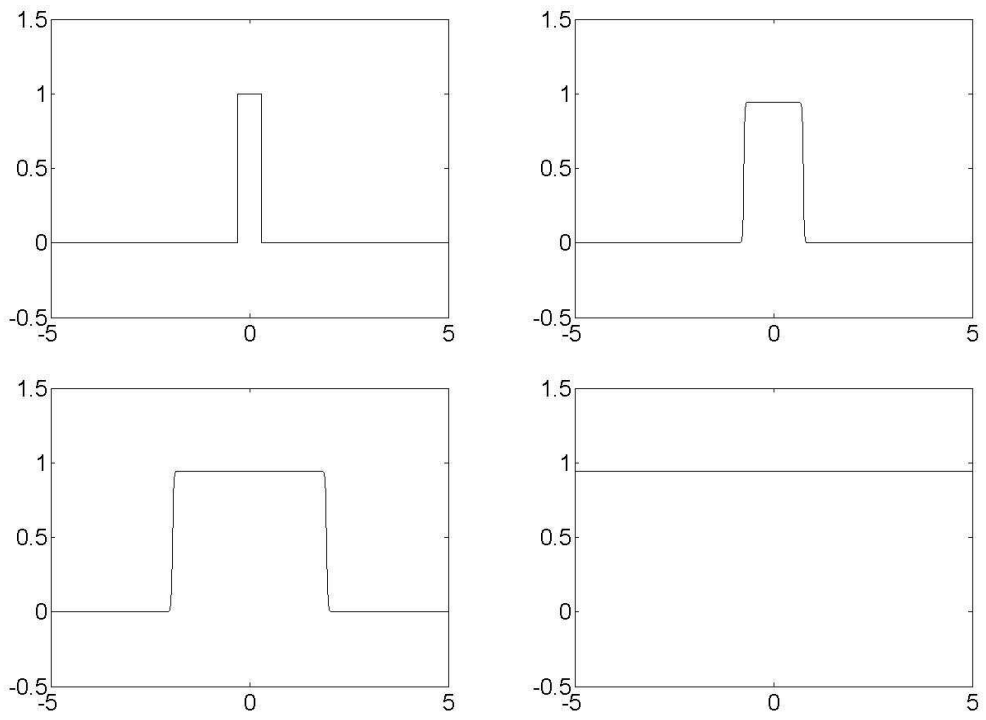

FIG. 4.7. The advective Allen-Cahn equation when $V$ is small, $V_{0}=10$, at time $t=0,0.1,0.2$, and 0.3 respectively.
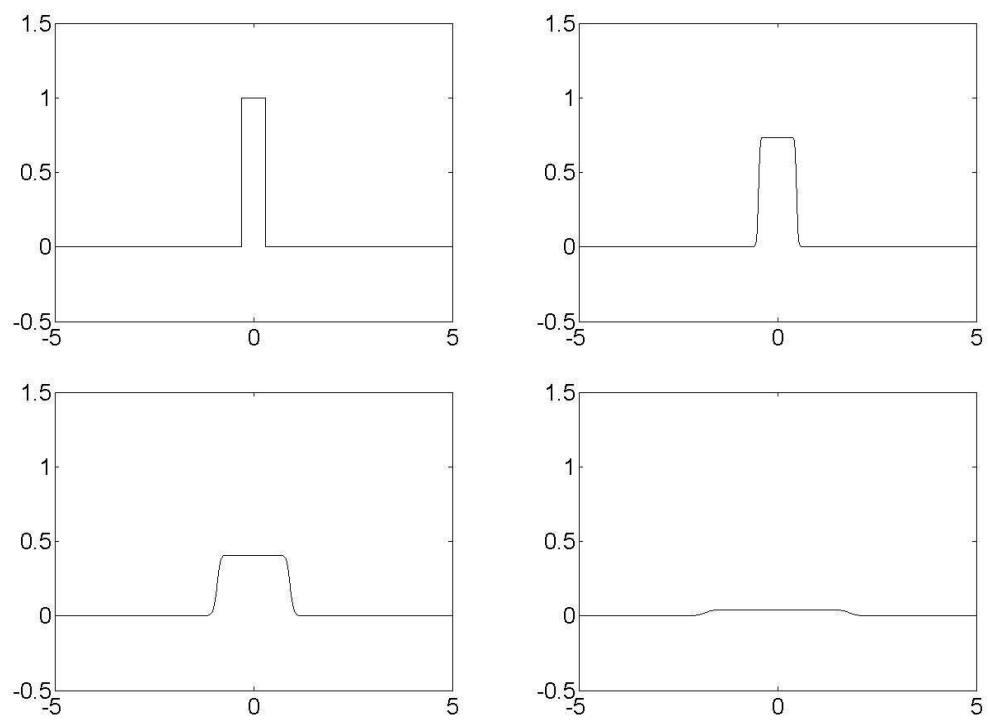

FIG. 4.8. The advective Allen-Cahn equation when $V$ is large, $V_{0}=30$, at time $t=0,0.1,0.2$, and 0.3 respectively.

and $\left|u_{\Delta x, \Delta t}(t)-u_{\Delta x / 2, \Delta t}(t)\right|$ in Figures 4.2 and 4.3 respectively. Here $u_{\Delta x, \Delta t}(t)$ is the solution at time $t$ for grid size $\Delta x$ and time step $\Delta t$. In both of these figures, we solve for 1 dimension advective Cahn-Hilliard equation where $\vec{V}=600 x, \epsilon=0.01$, and $A=1$ in a $[-5,5]$ interval with initial value of $u(0)=\chi_{[-0.3,0.3]}$. We run the solution through $t=0.002$. 

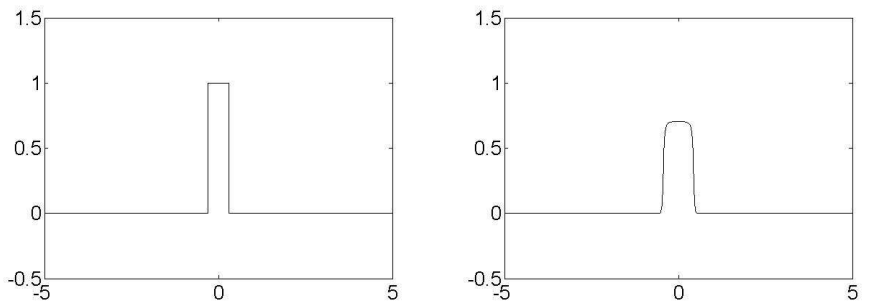

Fig. 4.9. The advective nonlocal Allen-Cahn equation when $V$ is small, $V_{0}=3.0$, at time $t=0$ and 0.2 respectively.
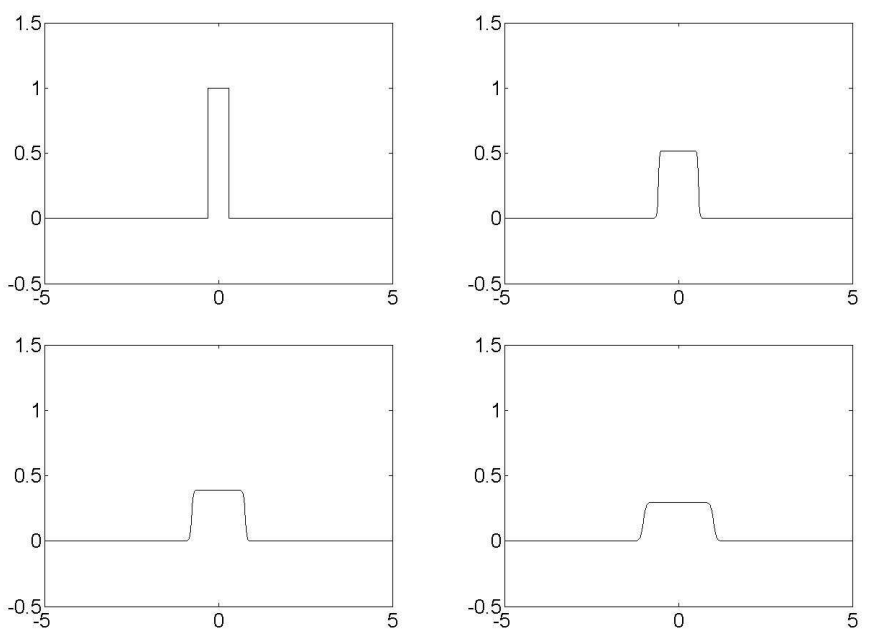

Fig. 4.10. The advective nonlocal Allen-Cahn equation when $V$ is large, $V_{0}=10$, at time $t=0$, $0.1,0.2$, and 0.3 respectively.

4.2. 1D result: the advective Cahn-Hilliard equation. We begin from the basic $1 \mathrm{D}$ case where $u(x, 0)=\chi_{[-\alpha, \alpha]}$ and $\vec{V}=V_{0} x$. The value of $V_{0}$ is tuned to show different types of solutions. The parameter $\epsilon$ is taken to be 0.01 , and $g(u)=u^{2}(1-u)^{2}$. $\alpha$ is taken as 0.3 . We run the simulation on the interval $[-5,5]$ with 2048 grid points. The time step is taken to be $\Delta t=1 \times 10^{-6}$, with 5000 time steps in total. The result of the advective Cahn-Hilliard Equation (1.1) contains two different types of solutions when $\vec{V}$ changes. When $\vec{V}$ is small, the solution develops a dimple in the middle, then stops, and does not break up further. When $\vec{V}$ is large, the solution eventually breaks up, and the smaller droplets continue to move apart; see Figure 4.4 and Figure 4.5.

The threshold value of $V_{0}$ is drawn on Figure 4.6, depending on the initial size of the droplet. The curve is an inverse quadratic curve of $V_{0} M^{2}=1.326$, which fits the prediction of Equation (2.18).

4.3. 1D result: the advective Allen-Cahn equation. As $\vec{V}$ increases, two different types of result appear for Equation (1.2). When $\vec{V}$ is small, the solution develops towards a constant given by the solution of $V_{0} u+\frac{1}{\epsilon} f(u)=0$. When $\vec{V}$ is large and the above equation does not have a solution, the solution expands and decreases 

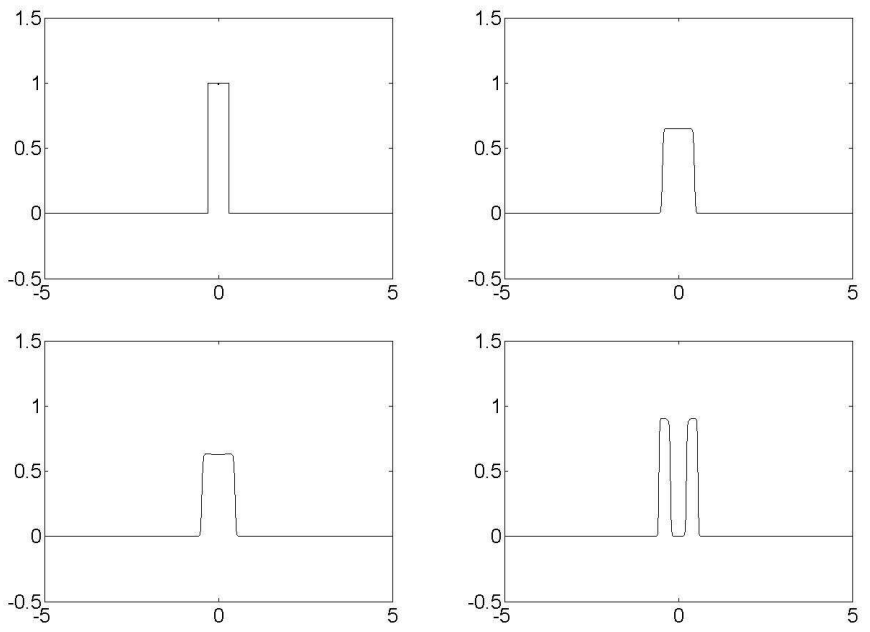

FIG. 4.11. The advective nonlocal Allen-Cahn equation when the initial value has an insubstantial dent near the origin, $V_{0}=5.0$, at $t=0,0.25$, 0.35, and 0.45 respectively.
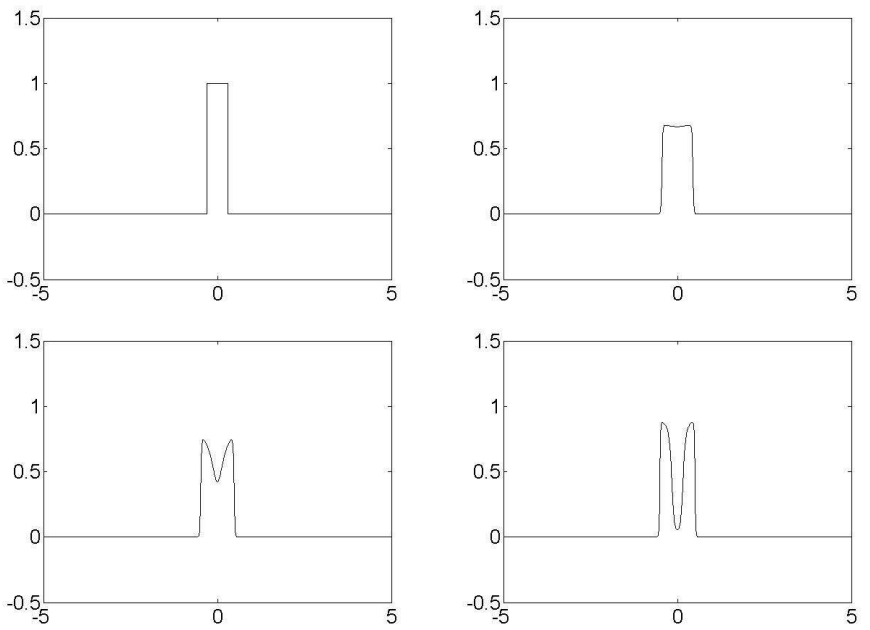

FIG. 4.12. The advective nonlocal Allen-Cahn equation when $V$ is not linear, see text for the formula of $V$, at $t=0,0.2,0.25$ and 0.30 respectively.

towards zero. The threshold is not related to $\alpha$ at all; see Figure 4.7 and Figure 4.8. Most numerical parameters are the same as that of the Cahn-Hilliard case: $\alpha$ is taken as 0.3 , the simulation is on the interval $[-5,5]$ with 2048 grid points. $\epsilon=0.01$. The difference is in the time step and the strength of velocity field. In the graphs shown, $\Delta t=0.001$, and the values of $V_{0}$ are 10.0 and 30.0 respectively.

4.4. 1D result: the advective nonlocal Allen-Cahn equation. Under the same setting, the advective nonlocal Allen-Cahn Equation (1.3) has two different types of results when $V_{0}$ changes. The threshold values of $V_{0}$ are listed in Table 4.4. When $\Omega$ is smaller, these two thresholds also decrease. When $\vec{V}$ is small, the solution 


\begin{tabular}{|c|c|}
\hline Value of $\alpha$ & Threshold of $V_{0}$ \\
\hline 0.5 & 7 \\
\hline 0.2 & 18 \\
\hline
\end{tabular}

TABLE 4.1. Threshold for the advective nonlocal Allen-Cahn equation

decreases and settles into a non-constant steady state depicting a single droplet. When $\vec{V}$ is large, the solution decays to a small constant consistent with mass conservation; see Figure 4.9 and Figure 4.10. The numerical parameters are the same as in the previous subsection. $\alpha$ is taken as 0.3 . The simulation is on interval $[-5,5]$ with 2048 grid points. $\epsilon=0.01$. Time step $\Delta t=0.001$, and the values of $V_{0}$ in the results shown are 3.0 and 10.0 respectively.

This represents a typical Allen-Cahn solution that does not show droplet breakup. The reason comes from the maximum principle, which was explained in Theorem 3.5. However, if the initial value is non-monotone, things become different. Even a small concavity at the origin leads to a completely different evolution. In Figure 4.11 we take $\vec{V}(x)=5 x$, but the initial value is taken as 1 in $[-0.5,-0.01) \cup(0.01,0.5], 0.99$ in $[-0.01,0.01]$, and 0 otherwise. The solution shows a breakup.

Another situation of droplet breakup involves a different velocity field $\vec{V}$. Figure 4.12 is the result for the case when $V=V_{0}\left(x-\frac{1}{100} x^{2}\right), V_{0}=5.0$ where $x \geq 0$ and expanded as an odd function to $x<0$. Note that this velocity field does not satisfy the condition of Theorem 3.6; see Figure 4.12.

4.5. 2D result. Since the $1 \mathrm{D}$ case shows interesting results, it is natural to perform simulations in higher dimensions where we have additional geometry. We tried two different cases for $2 \mathrm{D}$ results, respectively under an expanding velocity field and a sheer flow. The velocity field is prescribed as

$$
\vec{V}(x, y)=\left(V_{0} x, V_{0} y\right)
$$

for the expanding case, where $V_{0}$ is 2000 for the advective Cahn-Hilliard equation cases and 10 for the advective nonlocal Allen-Cahn equation cases. The velocity field is

$$
\vec{V}(x, y)=\left(0,-V_{0} x\right)
$$

for the sheer flow, where $V_{0}$ is 10000 for the advective Cahn-Hilliard equation and 100 for the advective nonlocal Allen-Cahn equation. The advective Cahn-Hilliard equation and the advective nonlocal Allen-Cahn equation are both tested for these cases. For all cases, we solve the equation in the region $[-1,1] \times[-1,1]$ with $128 \times 128$ mesh size, and $\epsilon=0.01$. For the expanding flow, we test two cases with different initial values. The initial value for the first case is 1 on $[-0.3,0.3] \times[-0.3,0.3]$ and 0 otherwise. In the second case the initial value is 1 on a circle of radius 0.3 and 0 otherwise. For the sheer flow, the initial value is 1 on $[-0.1,0.1] \times[-0.1,0.1]$ and 0 otherwise.

The time step is $1 \times 10^{-6}$ for the advective Cahn-Hilliard equation and $1 \times 10^{-4}$ for the advective Allen-Cahn equation. These parameters are chosen to emphasize the difference in their breakup phenomena; see Figure 4.13 to Figure 4.18.

Similar to the 1D case, the advective Cahn-Hilliard equation has a droplet breakup, while the advective nonlocal Allen-Cahn equation does not. Comparatively, 


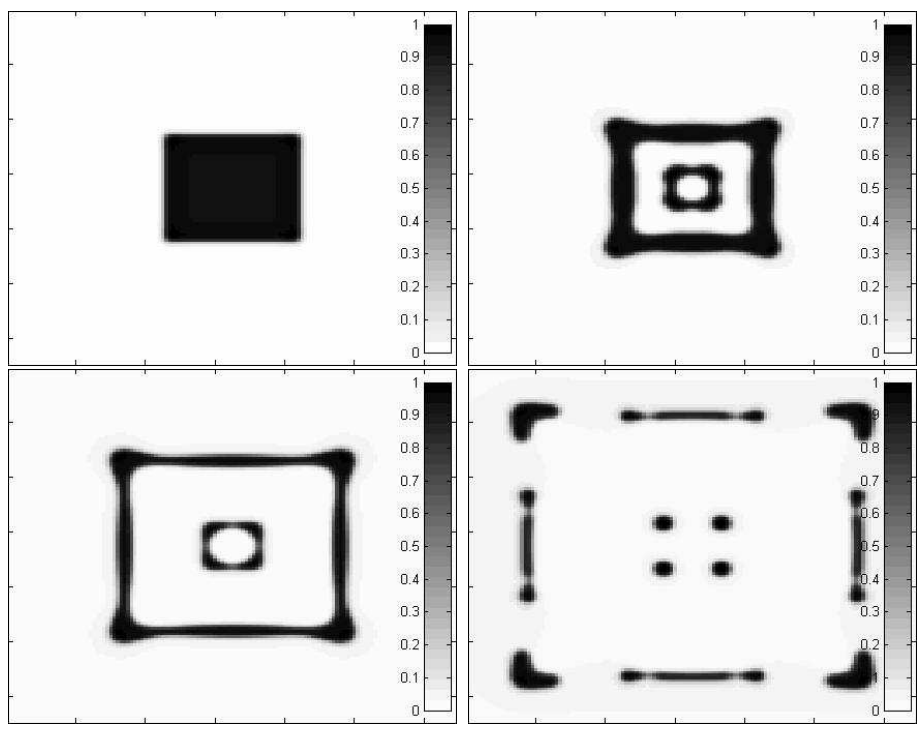

FIG. 4.13. The advective Cahn-Hilliard equation breakup under a $2 D$ expanding flow with a square initial value at $t=0,2.5 \times 10^{-4}, 5.0 \times 10^{-4}, 7.5 \times 10^{-4}$ respectively. $V_{0}=2000$.

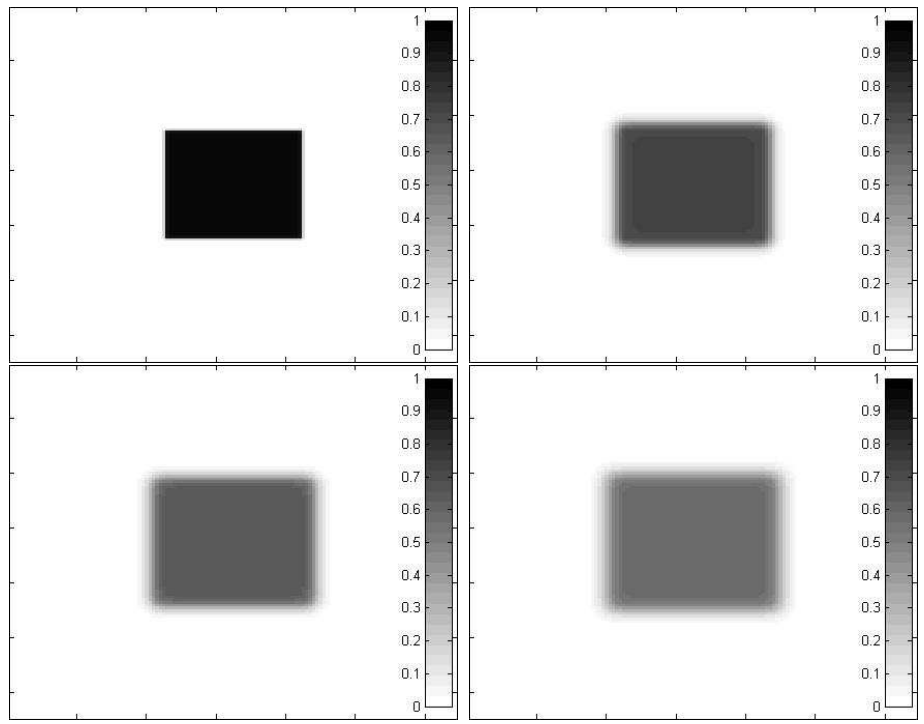

FIG. 4.14. The advective nonlocal Allen-Cahn equation result under a $2 D$ expanding flow with a square initial value at $t=0,0.02,0.04,0.06$ respectively. $V_{0}=10$.

the Cahn-Hilliard model shows a surface tension based breakup while Allen-Cahn model fails to do so in all cases.

4.6. 3D result. For the $3 \mathrm{D}$ case, we used a parallel machine in the National Energy Research Scientific Computing Center (NERSC) to solve the problem. Due to the complexity of the problem, an operator splitting scheme is used. Instead of 


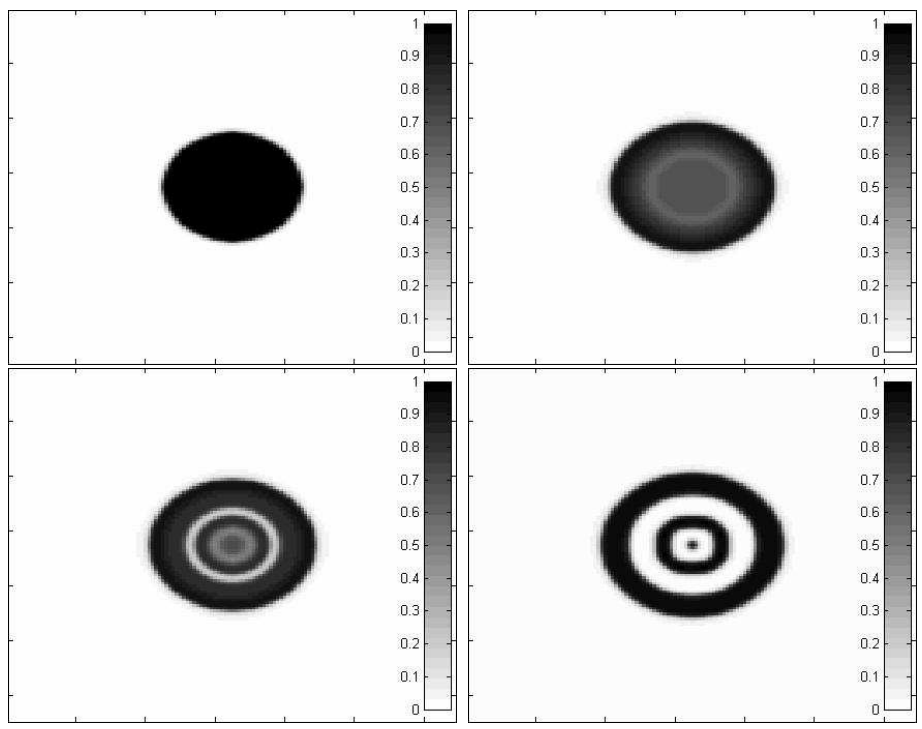

FIG. 4.15. The advective Cahn-Hilliard equation breakup under a $2 D$ expanding flow with radially symmetric initial value at $t=0,1.5 \times 10^{-4}, 1.7 \times 10^{-4}, 2.0 \times 10^{-4}$ respectively. $V_{0}=2000$.

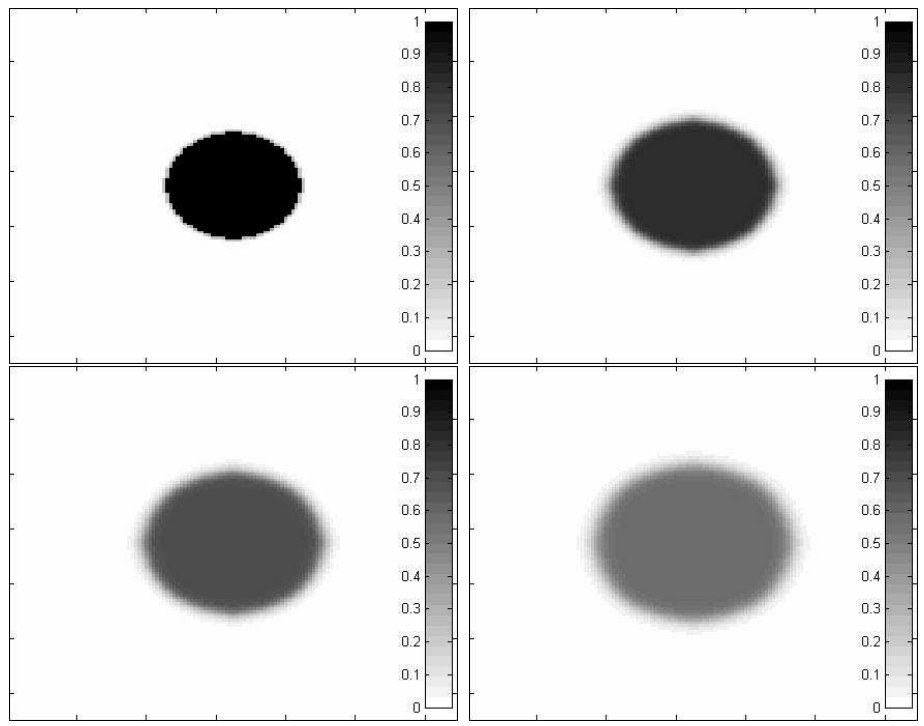

FIG. 4.16. The advective nonlocal Allen-Cahn equation result under a $2 D$ expanding flow with radially symmetric initial value at $t=0,0.02,0.04,0.06$ respectively. $V_{0}=10$.

solving Equation (4.1) directly, every time step is split into an advection step

$$
\frac{u^{*}-u^{n}}{\Delta t}+\nabla \cdot\left(u^{n} \vec{V}\right)=0,
$$

and Cahn-Hilliard (or Allen-Cahn, respectively) step

$$
\frac{u^{n+1}-u^{*}}{\Delta t}=-\Delta\left(\epsilon \Delta\left(A u^{n+1}+(1-A) u^{*}\right)-\frac{1}{\epsilon} f\left(u^{*}\right)\right) .
$$




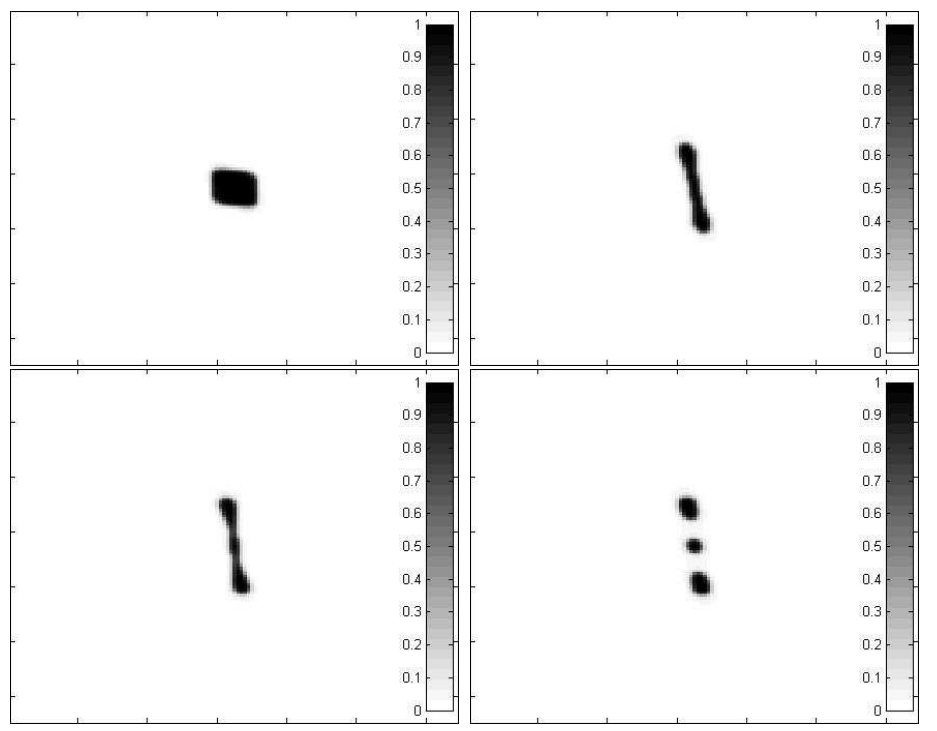

FIG. 4.17. The advective Cahn-Hilliard equation breakup under a $2 D$ sheer flow at $t=0,0.0008$, 0.001 and 0.0012 respectively. $V_{0}=10000$.

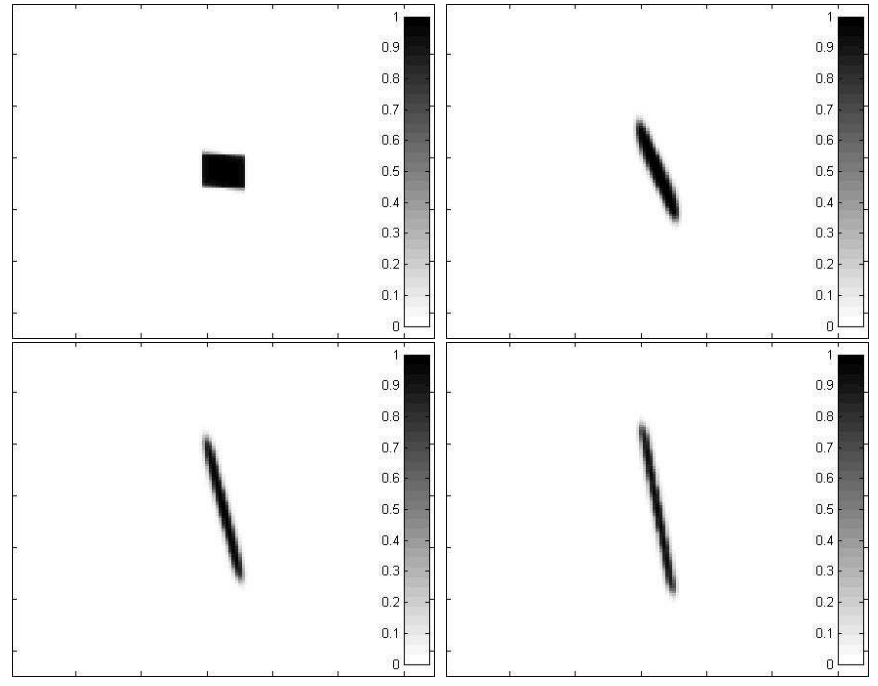

FIG. 4.18. The advective nonlocal Allen-Cahn equation result under a $2 D$ sheer flow at $t=0$, $0.02,0.04$ and 0.06 respectively. $V_{0}=100$.

The operator splitting and advection step are done by an ALE-AMR code [23]. The Cahn-Hilliard step is solved by a specifically written finite element package.

The simulation is run on a $[0,1]^{3}$ grid, with initial value being 1 on $[0.35,0.65]^{3}$ and 0 elsewhere. $\epsilon$ is still 0.01 . The velocity field is prescribed as

$$
\vec{V}(x, y, z)=\left(V_{0}(x-0.5), V_{0}(y-0.5), V_{0}(z-0.5)\right) .
$$

where $V_{0}=10000.0$. The time step is $2 \times 10^{-7}$, and $\epsilon=0.01$. 

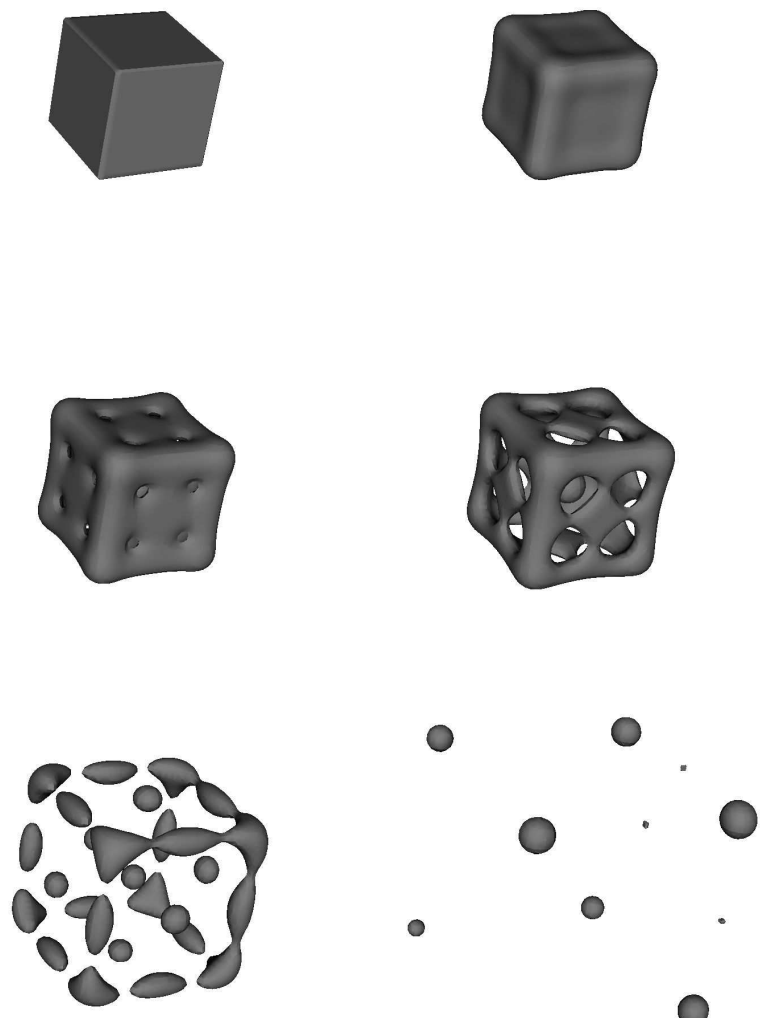

0

FIG. 4.19. The advective Cahn-Hilliard equation breakup under a $3 D$ expanding flow at time $t=0,0.3 \times 10^{-4}, 0.4 \times 10^{-4}, 0.5 \times 10^{-4}, 0.8 \times 10^{-4}$, and $1.5 \times 10^{-4}$ respectively.
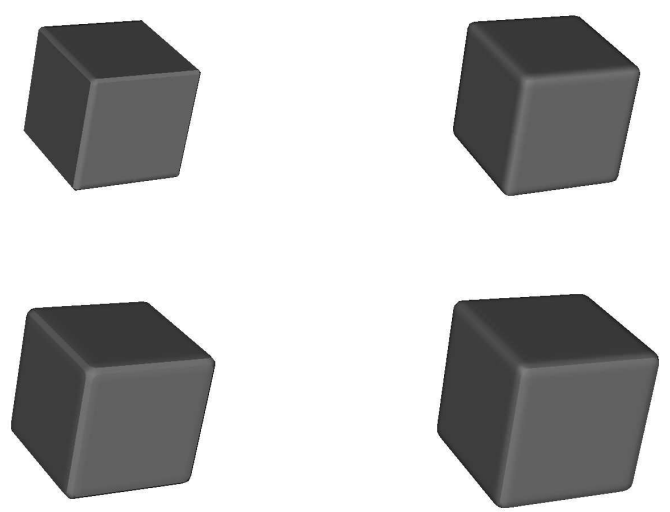

FIG. 4.20. The advective nonlocal Allen-Cahn equation's result under a $3 D$ expanding flow at time $t=0,0.5 \times 10^{-5}, 1.0 \times 10^{-5}$, and $1.5 \times 10^{-5}$ respectively. 


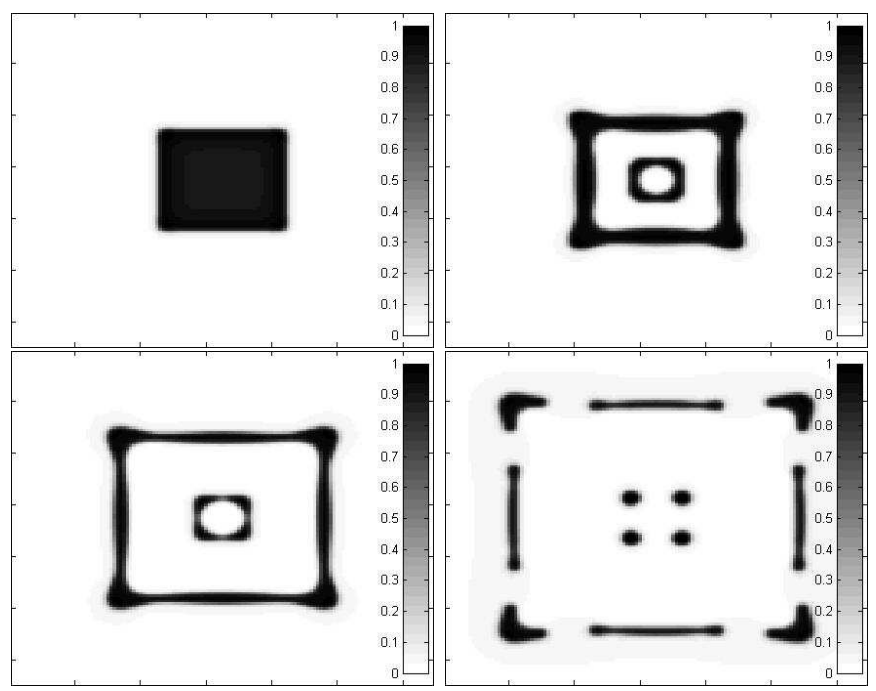

FIG. 4.21. The advective Cahn-Hilliard equation breakup under a $2 D$ expanding flow with noise of strength 0.01 in the initial value. It has a similar structure to that without noise. $V_{0}=2000$, snapshots are taken at time $t=0,2.5 \times 10^{-4}, 5.0 \times 10^{-4}$ and $7.5 \times 10^{-4}$ respectively.

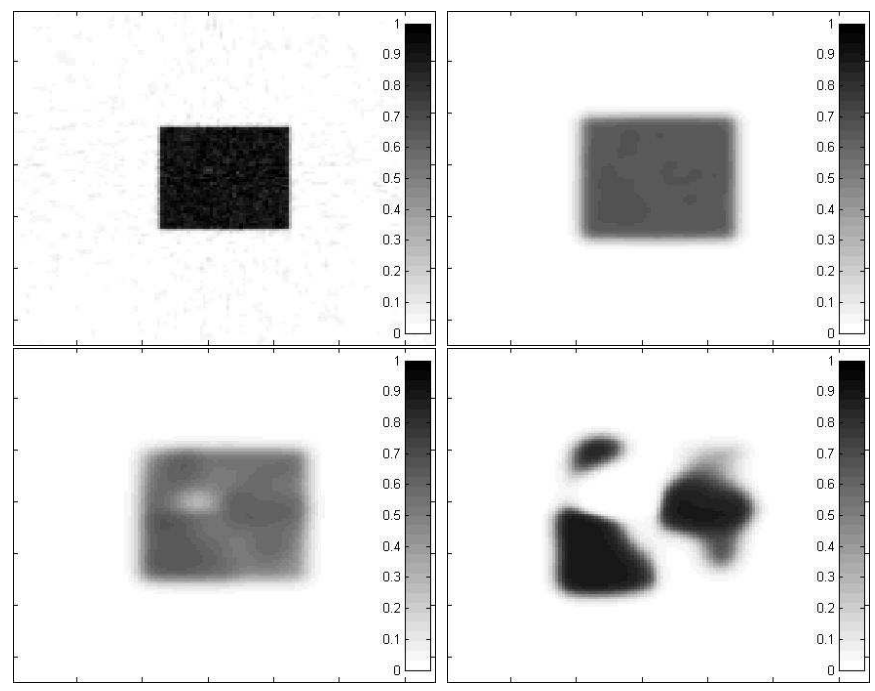

FIG. 4.22. The advective nonlocal Allen-Cahn equation breakup under a $2 D$ expanding flow with noise of strength 0.01 in the initial value. Without noise, it will not break up. $V_{0}=10$, snapshots are taken at $t=0,0.02,0.04,0.06$ respectively.

The advective Cahn-Hilliard equation has a droplet breakup similar to that of $2 \mathrm{D}$ case. The advective nonlocal Allen-Cahn equation simply performs a droplet expansion and then merges into the background or stops expanding, depending on the velocity field and droplet size; see Figure 4.19 and Figure 4.20.

4.7. Noise. The advective Allen-Cahn equation is more susceptible to noise compared to the advective Cahn-Hilliard equation. For the advective Allen-Cahn 


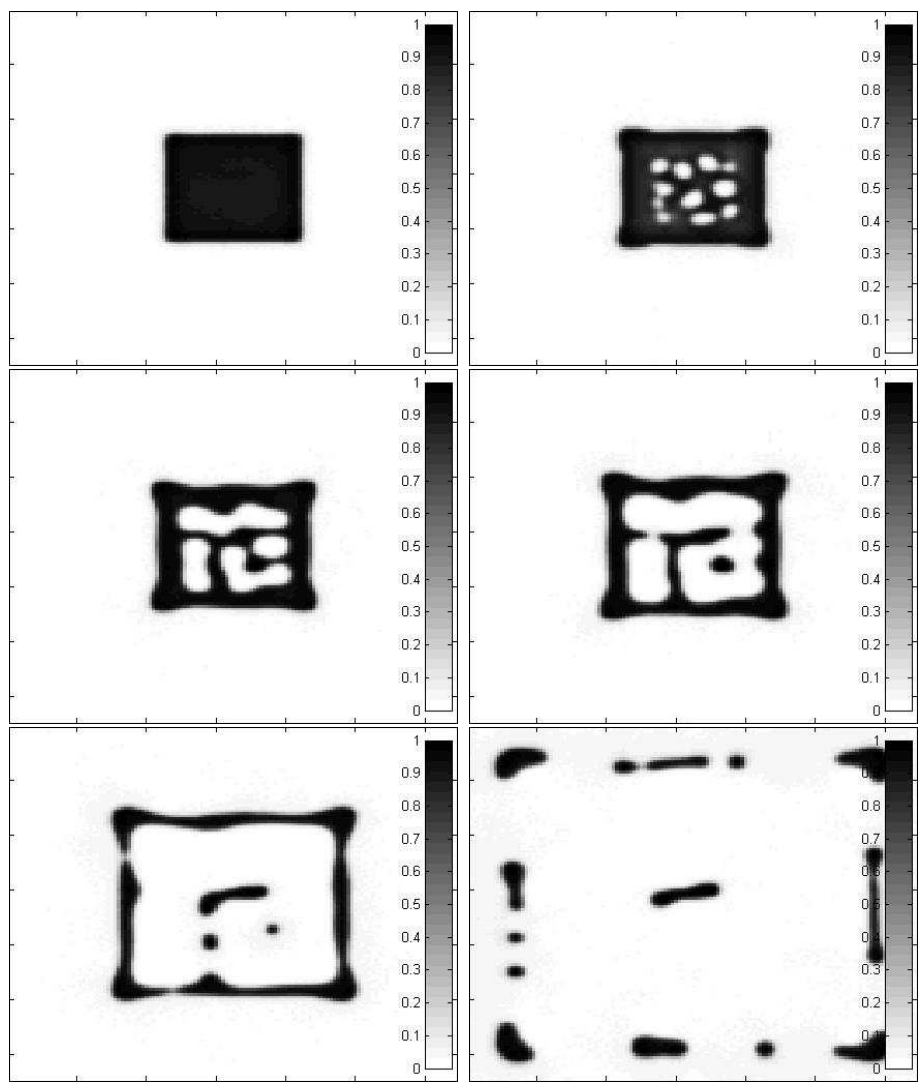

FIG. 4.23. The advective Cahn-Hilliard equation breakup under a $2 D$ expanding flow with continual noise over time. Symmetry is broken under this noise strength. $V_{0}=2000$, snapshots are taken at time $t=0,1.0 \times 10^{-4}, 2.0 \times 10^{-4}, 3.0 \times 10^{-4}, 5.0 \times 10^{-4}$ and $8.0 \times 10^{-4}$ respectively.

equation, even small noise in the initial value would lead to quite different behavior in terms of droplet breakup. However, the advective Cahn-Hilliard equation requires much stronger noise, or noise over time to make the result change. With strong enough noise, the droplet breakup shows some irregularity and breaks symmetry. Figure 4.21 and 4.22 have the same setting as Figure 4.13 and 4.14, except for a Gaussian noise of strength 0.01 added on the initial value. Figure 4.23 and 4.24, on the other hand, adds a Gaussian noise every time step.

\section{Conclusion}

In this paper we focus on the properties and numerical simulation of the CahnHilliard and Allen-Cahn equations with advection of a prescribed compressible flow. We have shown existence and uniqueness properties, and breakup conditions for both equations. For the advective Cahn-Hilliard equation, the droplet breakup condition is studied using a formal asymptotic analysis. Breakup will happen when the velocity field is large enough, and the threshold strength varies inverse quadratically with droplet size. For the advective Allen-Cahn equation, the breakup condition is studied using a maximum principle analysis. Breakup will not happen in this case without some kind of perturbation. Numerical results are provided in one, two, and three space 

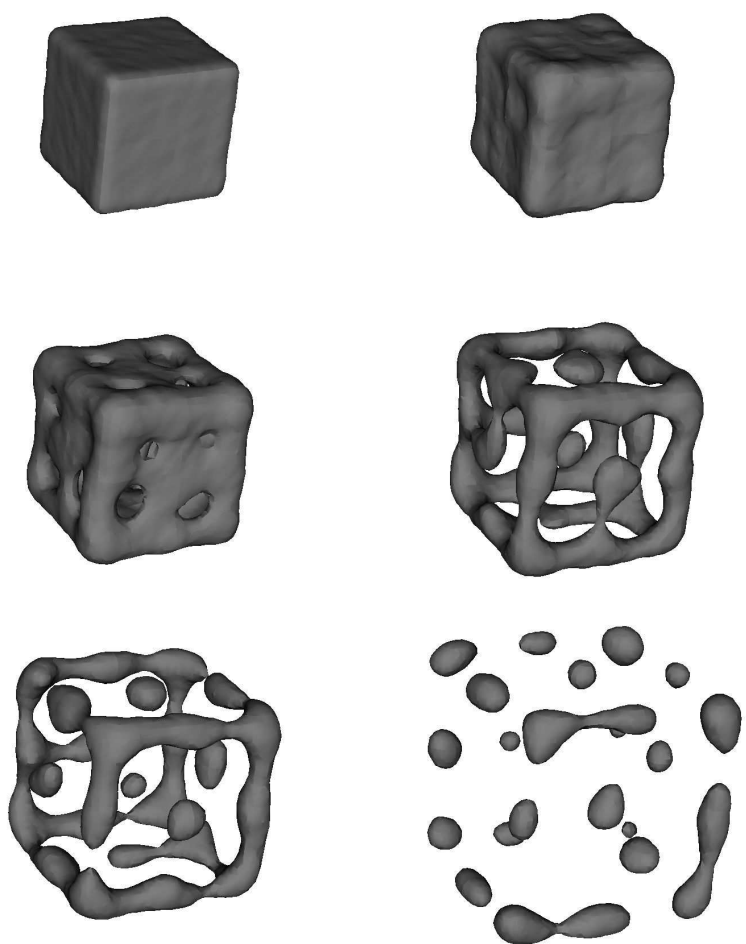

FIG. 4.24. The advective Cahn-Hilliard breakup under a $3 D$ expanding flow with continual noise over time at time $t=0,0.3 \times 10^{-4}, 0.4 \times 10^{-4}, 0.5 \times 10^{-4}, 0.8 \times 10^{-4}$, and $1.5 \times 10^{-4}$ respectively. Symmetry is broken under this noise strength.

dimensions, with various initial conditions and different kinds of background flow. We also test numerical simulations with noise. The theoretical breakup condition fits well with the numerical condition.

Eventually we need to simulate the droplet breakup phenomenon with surface tension. Thus, for the future work it is necessary to couple this model with other compressible fluid models. It is important to consider the impact of the phase field variable on the velocity field itself, and see how this model works within the full problem.

Acknowledgment. The idea to study these problems came from a series of discussions with David Eder of Lawrence Livermore National Lab and Alice Koniges of Lawrence Berkeley National Lab regarding suitable 'toy' problems on which to build a large scale simulator for plasmas, including the material in the National Ignition Facility, that might incorporate effects of surface tension dynamics as the material cools. Current computational models neglect surface tension, yet are used to study dynamics of particulates as they break up in the chamber. The model problems considered here are far from the final physics of such complex processes, yet they yield considerable quantitative and qualitative results that are useful for designing and testing code and for designing more complex models. Such reduced modeling has 
a long history in applied mathematics including many works by David Levermore for whom this special issue is dedicated. We specifically want to thank Alice Koniges, Aaron Fisher and Kirsten Fagnan for their kind help in the usage of the ALE-AMR code.

This project is supported by University of California Lab Fees Research Grant. Theodore Kolokolnikov is supported by Natural Sciences and Engineering Research Council of Canada(NSERC) discovery grant 47050. The National Energy Research Scientific Computing Center(NERSC) provided computing resources for the numerical simulations in $3 \mathrm{D}$.

\section{REFERENCES}

[1] H. Abels and E. Feireisl, On a diffuse interface model for a two-phase flow of compressible viscous fluids, Indiana Univ. Math. J., 57(2), 659-698, 2008.

[2] N.D. Alikakos, P.W. Bates, and X. Chen, Convergence of the Cahn-Hilliard equation to the Hele-Shaw model, Arch. Rational Mech. Anal., 128(2), 165-205, 1994.

[3] S. Allen and J. Cahn, A microscopic theory for antiphase boundary motion and its application to antiphase domain coarsening, Acta Metallurgica, 27, 1085-1095, 1979.

[4] M. Avellaneda and A. Majda, Mathematical models with exact renormalization for turbulent transport, Commun. Math. Phys., 131, 381-429, 1990. 10.1007/BF02161420.

[5] M. Avellaneda and A.J. Majda, Renormalization theory for eddy diffusivity in turbulent transport, Phys. Rev. Lett., 68(20), 3028-3031, May 1992.

[6] J. Ball, Remarks on the blow-up and nonexistence theorems for nonlinear evolution equations, Quart. J. Math. Oxford, 28, 473-486, 1977.

[7] J.W. Barrett, J.F. Blowey, and H. Garcke, Finite element approximation of a fourth order nonlinear degenerate parabolic equation, Numer. Math., 80(4), 525-556, 1998.

[8] A.L. Bertozzi, Heteroclinic orbits and chaotic dynamics in planar fluid flows, SIAM J. Math. Anal., 19, 1271-1294, 1988.

[9] A.L. Bertozzi, N. Ju, and H.W. Lu, A biharmonic modified forward time stepping method for fourth order nonlinear diffusion equations, Discr. Cont. Dyn. Sys., 29(4), 1367-1391, 2011.

[10] R. Bhagavatula, D. Jasnow, and T. Ohta, Nonequilibrium interface equations: An application to thermocapillary motion in binary systems, J. Stat. Phys., 88, 1013-1031, 1996.

[11] F. Boyer, Mathematical study of multi-phase flow under shear through order parameter formulation, Asymptot. Anal., 20(2), 175-212, 1999.

[12] J.W. Cahn and J.E. Hilliard, Free energy of a nonuniform system. i. Interfacial free energy, J. Chem. Phys., 28, 258-267, Feb. 1958.

[13] R. Caiden, R.P. Fedkiw, and C. Anderson, A numerical method for two-phase flow consisting of separate compressible and incompressible regions, J. Comput. Phys., 166(1), 1-27, 2001.

[14] R. Choksi and P. Sternberg, Periodic phase separation: The periodic Cahn-Hilliard andisoperimetric problems, Interfaces and Free Boundaries, 8, 371-392, 2006.

[15] E. Feireisl, H. Petzeltova, E. Rocca, and G. Schimperna, Analysis of a phase-field model for two-phase compressible fluids, Math. Models Methods Appl. Sci., 20(7), 1129-1160, 2010.

[16] J.B. Greer and A.L. Bertozzi, Traveling wave solutions of fourth order PDEs for image processing, SIAM. J. Math. Anal., 36(1), 38-68, 2004.

[17] Y. He, Y. Liu, and T. Tang, On large time-stepping methods for the Cahn-Hilliard equation, Appl. Numer. Math., 57(5-7), 616-628, 2007.

[18] D. Henry, Geometric Theory of Semilinear Parabolic Equations, Lecture Notes in Mathematics, Springer-Verlag, Berlin, 840, 1981.

[19] D. Jacqmin, Calculation of two-phase Navier-Stokes flows using phase-field modeling, J. Comput. Phys., 155(1), 96-127, 1999.

[20] B.S. Kerner and V.V. Osipov, Autosolitions: A New Approach to Problem of Self-Organization and Turbulence, Kluwer Academic Publishers, Dordrecht, 1994.

[21] J. Kim, K. Kang, and J. Lowengrub, Conservative multigrid methods for Cahn-Hilliard fluids, J. Comput. Phys., 193(2), 511-543, 2004.

[22] T. Kolokolnikov, M. Ward, and J. Wei, Self-replication of mesa patterns in reaction-diffusion models, Physica D: Nonlinear Phenomena, 236(2), 104-122, 2007.

[23] A.E. Koniges and et al., ALE-AMR: A new 3D multi-physics code for modeling laser/target effects, J. Phys., 244(032019), 2010. 
[24] C. Liu, N. Sato, and Y. Tonegawa, On the existence of mean curvature flow with transport term, Interfaces and Free Boundaries, 12, 251-277, 2010.

[25] J. Lowengrub and L. Truskinovsky, Quasi-incompressible Cahn-Hilliard fluids and topological transitions, R. Soc. Lond. Proc. Ser. A Math. Phys. Eng. Sci., 454(1978), 2617-2654, 1998.

[26] H.W. Lu, K. Glasner, A. Bertozzi, and C.J. Kim, A diffuse interface model for electrowetting droplets in a hele-shaw cell, J. Fluid Mechanics, 590, 411-435, 2007.

[27] Y. Maekawa, On a free boundary problem for viscous incompressible flows, Interfaces Free Bound., 9(4), 549-589, 2007.

[28] W. Mulder, S. Osher, and J. Sethian, Computing interface motion in compressible gas dynamics, J. Comput. Phys., 100, 209-228, 1992.

[29] J.C. Neu, The dynamics of a columnar vortex in an imposed strain, Phys. Fluids, 27, 23972402, 1984.

[30] Y. Nishiura and D. Ueyama, A skeleton structure of self-replicating dynamics, Physica D, 130(1), 73-104, 1999.

[31] R.L. Pego, Front migration in the nonlinear Cahn-Hilliard equation, Proc. Roy. Soc. London Ser. A, 422(1863), 261-278, 1989.

[32] J. Rubinstein and P. Sternberg, Nonlocal reaction-diffusion equations and nucleation, J. Appl. Math., 48(3), 249-264, 1992.

[33] M. Sussman and M. Ohta, A stable and efficient method for treating surface tension in incompressible two-phase flow, SIAM J. Sci. Comput., 31(4), 2447-2471, 2009.

[34] M. Sussman, P. Smereka, and S. Osher, A level set approach for computing solutions to incompressible two-phase flow, J. Comput. Phys., 114(1), 146-159, 1994.

[35] M. Sussman, K.M. Smith, M.Y. Hussaini, M. Ohta, and R. Zhi-Wei, A sharp interface method for incompressible two-phase flows, J. Comput. Phys., 221(2), 469-505, 2007.

[36] R. Temam, Infinite-Dimensional Dynamical Systems in Mechanics and Physics, Appl. Math. Sci., Springer-Verlag, New York, 68, 1988.

[37] R. Temam, Navier-Stokes Equations and Nonlinear Functional Analysis, CBMS-NSF Regional Conference Series in Applied Mathematics, SIAM, Philadelphia, PA, 2nd edition, 66, 1995.

[38] B.P. Vollmayr-Lee and A.D. Rutenberg, Fast and accurate coarsening simulation with an unconditionally stable time step, Phys. Rev. E, 68(6), 066703, 2003.

[39] C. Xu and T. Tang, Stability analysis of large time-stepping methods for epitaxial growth models, SIAM J. Numer. Anal., 44(4), 1759-1779, 2006.

[40] P. Yue, J.J. Feng, C. Liu, and J. Shen, Diffuse-interface simulations of drop coalescence and retraction in viscoelastic fluids, Journal of Non-Newtonian Fluid Mechanics, 129(3), 163176,2005 . 